Research Article

\title{
Bifurcation Analysis of a Discrete Food Chain Model with Harvesting
}

\author{
Abdul Qadeer Khan (iD) and Shahid Mehmood Qureshi \\ Department of Mathematics, University of Azad Jammu and Kashmir, Muzaffarabad 13100, Pakistan \\ Correspondence should be addressed to Abdul Qadeer Khan; abdulqadeerkhan1@gmail.com
}

Received 17 May 2021; Revised 10 August 2021; Accepted 20 August 2021; Published 14 October 2021

Academic Editor: Baogui Xin

Copyright (c) 2021 Abdul Qadeer Khan and Shahid Mehmood Qureshi. This is an open access article distributed under the Creative Commons Attribution License, which permits unrestricted use, distribution, and reproduction in any medium, provided the original work is properly cited.

We explore existence of fixed points, topological classifications around fixed points, existence of periodic points and prime period, and bifurcation analysis of a three-species discrete food chain model with harvesting. Finally, theoretical results are numerically verified.

\section{Introduction}

Many different types of interactions exist in nature between various species of organisms on this planet Earth and are studied under the discipline of ecology. Ecological interactions are most fundamental part in biology which determines community structure and development. Not all interactions are positive, some are negative also. One of the examples of negative correlation is ammensalism. Ammensalism is a type of ecological interactions between the members of two different species in which one is harmed, destroyed, or inhabited by the member of another species, while the other remains unaffected, neither harmed nor benefitted. It is a type of competitive behavior among different species and is frequently used to refer to asymmetrical competitive association. Research in the field of ecology draws the attention of several mathematicians such as Lotka [1] and Volterra [2]. Nowadays, ecologist and mathematician jointly contributed to the growth of this area of knowledge. Recently, many researchers have investigated the dynamical properties of discrete-time ecological models such as prey-predation, competitions, neutralism, and mutualism by studying fixed points, local and global attractivity, bounded, existence of bifurcation, and many more. For instance, Beddington et al. [3] have explored the behavior of following predator-prey model:

$$
\begin{aligned}
& x_{n+1}=x_{n} e^{r\left(1-\left(x_{n} / K\right)\right)-a y_{n},} \\
& y_{n+1}=\alpha x_{n}\left(1-e^{-a y_{n}}\right) .
\end{aligned}
$$

Chen [4] has explored global attractivity and permanence of the following discrete multispecies system:

$$
\begin{aligned}
& x_{i_{n+1}}=x_{i_{n}} e^{b_{i_{n}}-\sum_{l=1}^{k} a_{i l_{n}} x_{l_{n}}-\sum_{l=1}^{k} c_{i l_{n}} y_{l_{n}},} \\
& y_{j_{n+1}}=y_{j_{n}} e^{-r_{j_{n}}+\sum_{l=1}^{k} d_{j l_{n}} x_{l_{n}}-\sum_{l=1}^{k} e_{j l_{n}} y_{l_{n}} .}
\end{aligned}
$$

Fang and Chen [5] have explored the permanence of multispecies Lotka-Volterra competition predator-prey system with delays. Furthermore, Fang et al. [6] have explored the dynamics of the following system:

$$
\begin{aligned}
& x_{n+1}=x_{n} e^{a_{n}-b_{n} x_{n}-\left(c_{n} y_{n} /\left(m_{1 n}+m_{2 n} x_{n}+m_{3 n} y_{n}\right)\right)}, \\
& y_{n+1}=y_{n} e^{-d_{n}-e_{n} y_{n}+\left(f_{n} x_{n} /\left(m_{1 n}+m_{2 n} x_{n}+m_{3 n} y_{n}\right)\right)} .
\end{aligned}
$$

Agiza et al. [7] have explored chaotic dynamics of the following discrete model with Holling type II:

$$
\begin{aligned}
& x_{n+1}=a x_{n}\left(1-x_{n}\right)-\frac{b x_{n} y_{n}}{1+\varepsilon x_{n}}, \\
& y_{n+1}=\frac{d x_{n} y_{n}}{1+\varepsilon x_{n}} .
\end{aligned}
$$


Huo and Li [8] have explored stable periodic solution of the following discrete model:

$$
\begin{aligned}
& x_{n+1}=x_{n} e^{r_{1 n}-b_{1 n} x_{n}-a_{1 n} y_{n}}, \\
& y_{n+1}=y_{n} e^{r_{2 n}-a_{2 n}\left(y_{n} / x_{n}\right)} .
\end{aligned}
$$

Lu and Zhang [9] have studied the permanence and global attractivity of the following discrete system:

$$
\begin{aligned}
& x_{n+1}=x_{n} e^{a_{n}-b_{n} x_{n}-\left(m_{n} y_{n} /\left(A_{n}+x_{n}\right)\right)}, \\
& y_{n+1}=y_{n} e^{d_{n}-e_{n}\left(y_{n} / x_{n}\right)} .
\end{aligned}
$$

Zhao and Zhang [10] explored the chaos and permanence of the following discrete model:

$$
\begin{aligned}
& x_{n+1}=(1-d) x_{n} e^{r\left(1-\left(x_{n} / k\right)-a y_{n}\right)}+d \sigma x_{n-1} e^{r\left(1-\left(x_{n-1} / k\right)\right)}, \\
& y_{n+1}=(1-d) x_{n}\left(1-e^{-a y_{n}}\right) .
\end{aligned}
$$

Zhao et al. [11] have investigated the dynamics of the following discrete model:

$$
\begin{aligned}
& x_{n+1}=x_{n} e^{r\left(1-\left(x_{n} / k\right)\right)} f\left(y_{n}, y_{n-1}\right), \\
& y_{n+1}=x_{n}\left(1-f\left(y_{n}, y_{n-1}\right)\right),
\end{aligned}
$$

where $f\left(y_{n}, y_{n-1}\right)=e^{-a\left((1-d) y_{n}+d \sigma y_{n-1}\right)}$.

On the contrary, in recent years, many papers have been published to investigate the bifurcation analysis of certain discrete models by choosing step size as a bifurcation parameter. For example, Salman et al. [12] have explored bifurcation analysis of the following discrete system:

$$
\begin{aligned}
& x_{n+1}=x_{n}+\frac{\delta}{2}\left(x_{n}\left(1-x_{n}^{2}\right)-y_{n}\right), \\
& y_{n+1}=y_{n}+\delta y_{n}\left(-s+c x_{n}\right),
\end{aligned}
$$

by choosing step size $\delta$ as a bifurcation parameter. Liu and Xiao [13] have explored bifurcation analysis of the following discrete system:

$$
\begin{aligned}
& x_{n+1}=x_{n}+\delta\left(r x_{n}\left(1-x_{n}\right)-b x_{n} y_{n}\right), \\
& y_{n+1}=y_{n}+\delta\left(-d+b x_{n}\right) y_{n},
\end{aligned}
$$

by choosing step size $\delta$ as a bifurcation parameter. Hasan and Hama [14] have explored bifurcation analysis of the following discrete system:

$$
\begin{aligned}
& x_{n+1}=x_{n}+d x_{n}\left(1-x_{n}-\frac{y_{n}}{1+a x_{n}+b y_{n}}\right), \\
& y_{n+1}=y_{n}+d y_{n}\left(\frac{c x_{n}}{1+a x_{n}+b y_{n}}-e\right),
\end{aligned}
$$

by choosing step size $d$ as a bifurcation parameter. Wu and Zhang [15] have explored bifurcation analysis of the following discrete system:

$$
\begin{aligned}
& x_{n+1}=x_{n}+\delta x_{n}\left(K_{1}-\alpha_{1} x_{n}-\beta_{12} y_{n}-\gamma_{1} x_{n} y_{n}\right), \\
& y_{n+1}=y_{n}+\delta y_{n}\left(K_{2}-\alpha_{2} y_{n}-\beta_{21} x_{n}-\gamma_{2} x_{n} y_{n}\right),
\end{aligned}
$$

by choosing step size $\delta$ as a bifurcation parameter. Rana [16] has explored bifurcation analysis of the following discrete system:

$$
\begin{aligned}
& x_{n+1}=x_{n}+\delta\left(x_{n}\left(1-x_{n}\right)-\frac{a x_{n} y_{n}}{x_{n}+y_{n}}\right), \\
& y_{n+1}=x_{n}+\delta\left(-d y_{n}+\frac{b x_{n} y_{n}}{x_{n}+y_{n}}\right)
\end{aligned}
$$

by choosing step size $\delta$ as a bifurcation parameter. Rana and Kulsum [17] have explored bifurcation analysis of the following discrete system:

$$
\begin{aligned}
& x_{n+1}=x_{n}+\delta x_{n}\left(1-x_{n}-\frac{y_{n}}{x_{n}^{2}+a}\right), \\
& y_{n+1}=y_{n}+\delta y_{n}\left(\alpha-\frac{\beta y_{n}}{x_{n}}\right),
\end{aligned}
$$

by choosing step size $\delta$ as a bifurcation parameter. Motivated from the aforementioned studies, in this work, we explore existence of fixed points, topological classifications around fixed points, periodic points, and bifurcation analysis, by choosing step size $h$ as a bifurcation parameter, of the following three species discrete food chain model with harvesting:

$$
\begin{aligned}
& x_{n+1}=x_{n}+h\left(a_{1}\left(1-k_{1}\right) x_{n}-\alpha_{11} x_{n}^{2}\right), \\
& y_{n+1}=y_{n}+h\left(a_{2}\left(1-k_{2}\right) y_{n}-\alpha_{22} y_{n}^{2}-\alpha_{21} x_{n} y_{n}\right), \\
& z_{n+1}=z_{n}+h\left(a_{3} z_{n}-\alpha_{33} z_{n}^{2}-\alpha_{32} y_{n} z_{n}\right),
\end{aligned}
$$

which is a discrete form of the following model:

$$
\begin{aligned}
& \frac{\mathrm{d} x}{\mathrm{~d} t}=a_{1}\left(1-k_{1}\right) x-\alpha_{11} x^{2}, \\
& \frac{\mathrm{d} y}{\mathrm{~d} t}=a_{2}\left(1-k_{2}\right) y-\alpha_{22} y^{2}-\alpha_{21} x y, \\
& \frac{\mathrm{d} z}{\mathrm{~d} t}=a_{3} z-\alpha_{33} z^{2}-\alpha_{32} y z,
\end{aligned}
$$

by Euler forward formula, where $h$ is step size and $t$ is customary denoted by $n$. It is noted that, in model (16), $x(t)$, $y(t)$, and $z(t)$, respectively, denote populations of first, second, and third species. Moreover $z(t)$ denotes growth rate of first, second, and third species; $\alpha_{i i}(i=1,2,3)$ denotes the rate of decrease of first, second, and third species due to internal competitions; $\alpha_{21}$ denotes rate of decrease of second species due to attack of first species; $\alpha_{32}$ denotes the rate of decrease of third species due to attack of second species; $k_{1}$ and $k_{2}$, respectively, denote the harvesting rate of first and second species. It is also important to note that all parameters $h, a_{1}, a_{2}, a_{3}, k_{1}, k_{2}, \alpha_{11}, \alpha_{22}, \alpha_{21}, \alpha_{32}$, and $\alpha_{33}$ are positive. In addition, it is important here to mention that we 
will explore dynamical properties of the discrete-time model (15) instead of the continuous-time model, which is depicted in (16), because discrete-time models governed by difference equations are more realistic and appropriate than the continuous ones in the case where populations have nonoverlapping generations, and moreover, discrete models can also provide efficient computational results for numerical simulations [12, 13].

This paper is structured as follows. In Section 2, we study the existence of fixed points of model (15) algebraically. The linearized form of model (15) is presented in Section 3. In Section 4, we explored topological classification around fixed points of the model. Existence of periodic points of model (15) is explored in Section 5. In Section 6, we explored detailed analysis of bifurcation around fixed points of model (15). Theoretical results are verified numerically in Section 7. Brief summary of the paper is presented in Section 8.

\section{Study of Equilibrium Points}

Here, we will study the boundary and interior equilibrium points of model (15) as follows.

Lemma 1. Model (15) has atmost eight equilibrium points in $\mathbb{R}_{+}^{3}$. Precisely,

(i) $\forall h, a_{1}, a_{2}, a_{3}, k_{1}, k_{2}, \alpha_{11}, \alpha_{22}, \alpha_{21}, \alpha_{32}, \alpha_{33}>0$; model (15) has a trivial equilibrium point: $P_{1}=(0,0,0)$.

(ii) $\forall a_{3}, \alpha_{33}>0$; model (15) has boundary equilibrium point: $P_{2}=\left(0,0, a_{3} / \alpha_{33}\right)$.

(iii) $P_{3}=\left(0,\left(1-k_{2}\right) a_{2} / \alpha_{22}, 0\right)$ is a boundary equilibrium point of (15) if $k_{2}<1$.

(iv) $P_{4}=\left(\left(1-k_{1}\right) a_{1} / \alpha_{11}, 0,0\right)$ is a boundary equilibrium point of (15) if $k_{1}<1$.

(v) $P_{5}=\left(0,\left(1-k_{2}\right) a_{2} / \alpha_{22},\left(a_{3} \alpha_{22}-\alpha_{32}(1-\right.\right.$

$\left.\left.\left.k_{2}\right) a_{2}\right) / \alpha_{22} \alpha_{33}\right)$ is a boundary equilibrium point of (15) if $a_{3}>\alpha_{32}\left(1-k_{2}\right) a_{2} / \alpha_{22}$ with $k_{2}<1$.

(vi) $P_{6}=\left(\left(1-k_{1}\right) a_{1} / \alpha_{11}, 0, a_{3} / \alpha_{33}\right)$ is a boundary equilibrium point of (15) if $k_{1}<1$.

(vii) $P_{7}=\left(\left(1-k_{1}\right) a_{1} / \alpha_{11},\left(a_{2}\left(1-k_{2}\right) \alpha_{11}-a_{1}(1-\right.\right.$

$\left.\left.\left.k_{1}\right) \alpha_{21}\right) / \alpha_{11} \alpha_{22}, 0\right)$ is a boundary equilibrium point of (15) if $a_{2}>a_{1}\left(1-k_{1}\right) \alpha_{21} /\left(1-k_{2}\right) \alpha_{11}$ with $k_{1}, k_{2}<1$.

(viii) $P_{8}=\left(\left(1-k_{1}\right) a_{1} / \alpha_{11},\left(a_{2}\left(1-k_{2}\right) \alpha_{11}-a_{1}(1-\right.\right.$ $\left.\left.k_{1}\right) \alpha_{21}\right) / \alpha_{11} \alpha_{22},\left(a_{3} \alpha_{11} \alpha_{22}-a_{2}\left(1-k_{2}\right) \alpha_{11} \alpha_{32}+a_{1}(1-\right.$ $\left.\left.\left.k_{1}\right) \alpha_{21} \alpha_{32}\right) / \alpha_{11} \alpha_{22} \alpha_{33}\right)$ is an interior equilibrium point of

(15) if $k_{1}<1, \quad a_{2}>a_{1}\left(1-k_{1}\right) \alpha_{21} /\left(1-k_{2}\right) \alpha_{11}$ and $a_{3}>\left(a_{2}\left(1-k_{2}\right) \alpha_{11} \alpha_{32}-a_{1}\left(1-k_{1}\right) \alpha_{21} \alpha_{32}\right) / \alpha_{11} \alpha_{22}$.
Proof. If model (15) has an equilibrium point, $P=(x, y, z)$, then

$$
\begin{aligned}
& x=x+h\left(a_{1}\left(1-k_{1}\right) x-\alpha_{11} x^{2}\right), \\
& y=y+h\left(a_{2}\left(1-k_{2}\right) y-\alpha_{22} y^{2}-\alpha_{21} x y\right), \\
& z=z_{n}+h\left(a_{3} z-\alpha_{33} z^{2}-\alpha_{32} y z\right) .
\end{aligned}
$$

The simple computation yields that, for the values of $P_{i}(i=1, \ldots, 7),(17)$ satisfied identically. So, one can conclude that model (15) has seven boundary points: $P_{i}(i=1, \ldots, 7)$. In order to find interior point, from (17), one obtains

$$
\begin{aligned}
a_{1}\left(1-k_{1}\right)-\alpha_{11} x & =0, \\
a_{2}\left(1-k_{2}\right)-\alpha_{22} y-\alpha_{21} x & =0, \\
a_{3}-\alpha_{33} z-\alpha_{32} y & =0 .
\end{aligned}
$$

From $1^{\text {st }}$ of $(18)$, one obtains

$$
x=\frac{\left(1-k_{1}\right) a_{1}}{\alpha_{11}} .
$$

From $2^{\text {nd }}$ equation of (18) and (19), one obtains

$$
y=\frac{a_{2}\left(1-k_{2}\right) \alpha_{11}-a_{1}\left(1-k_{1}\right) \alpha_{21}}{\alpha_{11} \alpha_{22}} .
$$

From $3^{\text {rd }}$ equation of (18) and (20), one obtains

$$
z=\frac{a_{3} \alpha_{11} \alpha_{22}-a_{2}\left(1-k_{2}\right) \alpha_{11} \alpha_{32}+a_{1}\left(1-k_{1}\right) \alpha_{21} \alpha_{32}}{\alpha_{11} \alpha_{22} \alpha_{33}} .
$$

From (19)-(21), one can conclude that $P_{8}$ is an interior equilibrium point of (15) if $k_{1}<1, a_{2}>\left(a_{1}\left(1-k_{1}\right) \alpha_{21} /\right.$ $\left.\left(1-k_{2}\right) \alpha_{11}\right)$, and $a_{3}>\left(a_{2}\left(1-k_{2}\right) \alpha_{11} \alpha_{32}-a_{1}\left(1-k_{1}\right) \alpha_{21} \alpha_{32}\right)$ $/ \alpha_{11} \alpha_{22}$.

\section{Linearized Form of Model (15)}

The variational matrix $\left.J\right|_{P}$ about $P$ under the map:

$$
\left(f_{1}, f_{2}, f_{3}\right) \mapsto\left(x_{n+1}, y_{n+1}, z_{n+1}\right),
$$

where

$$
\begin{aligned}
& f_{1}=x+h\left(a_{1}\left(1-k_{1}\right) x-\alpha_{11} x^{2}\right) \\
& f_{2}=y+h\left(a_{2}\left(1-k_{2}\right) y-\alpha_{22} y^{2}-\alpha_{21} x y\right), \\
& f_{3}=z+h\left(a_{3} z-\alpha_{33} z^{2}-\alpha_{32} y z\right)
\end{aligned}
$$

is

$$
\left.J\right|_{P}=\left(\begin{array}{ccc}
1+h\left(a_{1}\left(1-k_{1}\right)-2 \alpha_{11} x\right) & 0 & 0 \\
-h \alpha_{21} y & 1+h\left(a_{2}\left(1-k_{2}\right)-2 \alpha_{22} y-\alpha_{21} x\right) & 0 \\
0 & -h \alpha_{32} z & 1+h\left(a_{3}-2 \alpha_{33} z-\alpha_{32} y\right)
\end{array}\right),
$$


with

$$
\begin{aligned}
& \lambda_{1}=1+h\left(a_{1}\left(1-k_{1}\right)-2 \alpha_{11} x\right), \\
& \lambda_{2}=1+h\left(a_{2}\left(1-k_{2}\right)-2 \alpha_{22} y-\alpha_{21} x\right), \\
& \lambda_{3}=1+h\left(a_{3}-2 \alpha_{33} z-\alpha_{32} y\right) .
\end{aligned}
$$

\section{Dynamical Behavior: Topological Properties of Equilibrium Points}

The dynamical behavior about fixed points $P_{i}(i=1, \ldots, 8)$ of model (15) is explored in this section.

4.1. Dynamical Behavior about $P_{1}$. From (25), eigenvalues of $J P_{P_{1}}$ about $P_{1}$ are

$$
\begin{aligned}
& \lambda_{1}=1+h a_{1}\left(1-k_{1}\right), \\
& \lambda_{2}=1+h a_{2}\left(1-k_{2}\right), \\
& \lambda_{3}=1+h a_{3} .
\end{aligned}
$$

The dynamical behavior about $P_{1}$ of model (15) is concluded as follows.

\section{Lemma 2.}

(i) For all allowed parametric values, $h, a_{1}, a_{2}, a_{3}, k_{1}, k_{2}$, $\alpha_{11}, \alpha_{22}, \alpha_{21}, \alpha_{32}, \alpha_{33}>0, P_{1}$ is not sink.

(ii) $P_{1}$ is a source if

$$
0<h<\min \left\{\frac{2}{a_{1}\left(k_{1}-1\right)}, \frac{2}{a_{2}\left(k_{2}-1\right)}\right\} .
$$

(iii) $P_{1}$ is a saddle if

$$
h>\max \left\{\frac{2}{a_{1}\left(k_{1}-1\right)}, \frac{2}{a_{2}\left(k_{2}-1\right)}\right\} .
$$

(iv) $P_{1}$ is nonhyperbolic if

$$
h=\frac{2}{a_{1}\left(k_{1}-1\right)},
$$

or

$$
h=\frac{2}{a_{2}\left(k_{2}-1\right)} \text {. }
$$

4.2. Dynamical Behavior about $P_{2}$. From (25), eigenvalues of $\left.J\right|_{P_{2}}$ about $P_{2}$ are

$$
\begin{aligned}
& \lambda_{1}=1+h a_{1}\left(1-k_{1}\right), \\
& \lambda_{2}=1+h a_{2}\left(1-k_{2}\right), \\
& \lambda_{3}=1-h a_{3} .
\end{aligned}
$$

The dynamical behavior about $P_{2}$ is concluded as follows:
Lemma 3. (i) $P_{2}$ is a sink if

$$
h>\max \left\{\frac{2}{a_{1}\left(k_{1}-1\right)}, \frac{2}{a_{2}\left(k_{2}-1\right)}\right\}, \quad 0<h<\frac{2}{a_{3}} .
$$

(ii) $P_{2}$ is a source if

$$
0<h<\min \left\{\frac{2}{a_{1}\left(k_{1}-1\right)}, \frac{2}{a_{2}\left(k_{2}-1\right)}\right\}, \quad h>\frac{2}{a_{3}} .
$$

(iii) $P_{2}$ is a saddle if

$$
h<\min \left\{\frac{2}{a_{1}\left(k_{1}-1\right)}, \frac{2}{a_{2}\left(k_{2}-1\right)}\right\}, \quad 0<h<\frac{2}{a_{3}} \text {. }
$$

(iv) $P_{2}$ is nonhyperbolic if

$$
h=\frac{2}{a_{3}},
$$

or

$$
h=\frac{2}{a_{1}\left(k_{1}-1\right)},
$$

or

$$
h=\frac{2}{a_{2}\left(k_{2}-1\right)} \text {. }
$$

4.3. Dynamical Behavior about $P_{3}$. From (25), eigenvalues of $\left.J\right|_{P_{3}}$ about $P_{3}$ are

$$
\begin{aligned}
& \lambda_{1}=1+h a_{1}\left(1-k_{1}\right), \\
& \lambda_{2}=1-h a_{2}\left(1-k_{2}\right), \\
& \lambda_{3}=1+h\left(\frac{a_{3} \alpha_{22}-a_{2} \alpha_{32}\left(1-k_{2}\right)}{\alpha_{22}}\right) .
\end{aligned}
$$

The dynamical behavior about $P_{3}$ is concluded as follows.

\section{Lemma 4}

(i) $P_{3}$ is a sink if

$$
\begin{aligned}
& h>\max \left\{\frac{2}{a_{1}\left(k_{1}-1\right)}, \frac{2 \alpha_{22}}{a_{2} \alpha_{32}\left(1-k_{2}\right)-a_{3} \alpha_{22}}\right\}, \\
& 0<h<\frac{2}{a_{2}\left(1-k_{2}\right)} .
\end{aligned}
$$

(ii) $P_{3}$ is a source if

$$
\begin{aligned}
& 0<h<\min \left\{\frac{2}{a_{1}\left(k_{1}-1\right)}, \frac{2 \alpha_{22}}{a_{2} \alpha_{32}\left(1-k_{2}\right)-a_{3} \alpha_{22}}\right\}, \\
& h>\frac{2}{a_{2}\left(1-k_{2}\right)} .
\end{aligned}
$$


(iii) $P_{3}$ is a saddle if

$$
\begin{aligned}
& h>\max \left\{\frac{2}{a_{1}\left(k_{1}-1\right)}, \frac{2}{a_{2}\left(1-k_{2}\right)}\right\}, \\
& 0<h<\frac{2 \alpha_{22}}{a_{2} \alpha_{32}\left(1-k_{2}\right)-a_{3} \alpha_{22}} .
\end{aligned}
$$

(iv) $P_{3}$ is nonhyperbolic if

$$
h=\frac{2}{a_{2}\left(1-k_{2}\right)}
$$

or

$$
h=\frac{2}{a_{1}\left(k_{1}-1\right)},
$$

or

$$
h=\frac{2 \alpha_{22}}{a_{2} \alpha_{32}\left(1-k_{2}\right)-a_{3} \alpha_{22}} .
$$

4.4. Dynamical Behavior about $P_{4}$. From (25), eigenvalues of $\mathrm{J}_{P_{4}}$ about $P_{4}$ are

$$
\begin{aligned}
& \lambda_{1}=1-h a_{1}\left(1-k_{1}\right), \\
& \lambda_{2}=1+h a_{2}\left(1-k_{2}\right), \\
& \lambda_{3}=1+h a_{3} .
\end{aligned}
$$

The dynamical behavior about $P_{4}$ is concluded as follows.

\section{Lemma 5.}

(i) For all allowed parametric values, $h, a_{1}, a_{2}, a_{3}, k_{1}, k_{2}, \alpha_{11}, \alpha_{22}, \alpha_{21}, \alpha_{32}, \alpha_{33}>0, P_{4}$ is not sink.

(ii) $P_{4}$ is a source if

$$
h>\max \left\{\frac{2}{a_{1}\left(1-k_{1}\right)}, \frac{2}{a_{2}\left(k_{2}-1\right)}\right\} .
$$

(iii) $P_{4}$ is a saddle if

$$
0<h<\min \left\{\frac{2}{a_{1}\left(1-k_{1}\right)}, \frac{2}{a_{2}\left(k_{2}-1\right)}\right\} .
$$

(iv) $P_{4}$ is nonhyperbolic if

$$
h=\frac{2}{a_{1}\left(1-k_{1}\right)},
$$

or

$$
h=\frac{2}{a_{2}\left(k_{2}-1\right)} \text {. }
$$

4.5. Dynamical Behavior about $P_{5}$. From (25), eigenvalues of $\mathrm{JI}_{P_{5}}$ about $P_{5}$ are

$$
\begin{aligned}
& \lambda_{1}=1+h a_{1}\left(1-k_{1}\right), \\
& \lambda_{2}=1-h a_{2}\left(1-k_{2}\right), \\
& \lambda_{3}=1+h\left(-a_{3}+\frac{\alpha_{32}\left(1-k_{2}\right) a_{2}}{\alpha_{22}}\right) .
\end{aligned}
$$

The dynamical behavior about $P_{5}$ is concluded as follows.

Lemma 6. (i) $P_{5}$ is a sink if

$$
\begin{aligned}
& h>\max \left\{\frac{2}{a_{1}\left(k_{1}-1\right)}, \frac{2 \alpha_{22}}{a_{3} \alpha_{22}-a_{2} \alpha_{32}\left(1-k_{2}\right)}\right\}, \\
& 0<h<\frac{2}{a_{2}\left(1-k_{2}\right)} .
\end{aligned}
$$

(ii) $P_{5}$ is a source if

$$
\begin{aligned}
& 0<h<\min \left\{\frac{2}{a_{1}\left(k_{1}-1\right)}, \frac{2 \alpha_{22}}{a_{3} \alpha_{22}-a_{2} \alpha_{32}\left(1-k_{2}\right)}\right\}, \\
& h>\frac{2}{a_{2}\left(1-k_{2}\right)} .
\end{aligned}
$$

(iii) $P_{5}$ is a saddle if

$$
\begin{aligned}
& h>\max \left\{\frac{2}{a_{1}\left(k_{1}-1\right)}, \frac{2 \alpha_{22}}{a_{3} \alpha_{22}-a_{2} \alpha_{32}\left(1-k_{2}\right)}\right\}, \\
& h>\frac{2}{a_{2}\left(1-k_{2}\right)} .
\end{aligned}
$$

(iv) $P_{5}$ is nonhyperbolic if

$$
h=\frac{2}{a_{1}\left(k_{1}-1\right)}
$$

or

$$
h=\frac{a_{3} \alpha_{22}-2 \alpha_{22}}{a_{2} \alpha_{32}\left(1-k_{2}\right)}
$$

or

$$
h=\frac{2}{a_{2}\left(1-k_{2}\right)} \text {. }
$$

4.6. Dynamical Behavior about $P_{6}$. From (25), eigenvalues of $\left.J\right|_{P_{6}}$ about $P_{6}$ are

$$
\begin{aligned}
& \lambda_{1}=1-h a_{1}\left(1-k_{1}\right), \\
& \lambda_{2}=1+h\left(a_{2}\left(1-k_{2}\right)-\frac{a_{1} \alpha_{21}\left(1-k_{1}\right)}{\alpha_{11}}\right), \\
& \lambda_{3}=1-h a_{3} .
\end{aligned}
$$


The dynamical behavior about $P_{6}$ is concluded as follows.

\section{Lemma 7.}

(i) $P_{6}$ is a sink if

$$
\begin{aligned}
& 0<h<\min \left\{\frac{2}{a_{1}\left(1-k_{1}\right)}, \frac{2}{a_{3}}\right\} \\
& h>\frac{2 \alpha_{11}}{a_{1} \alpha_{21}\left(1-k_{1}\right)-a_{2} \alpha_{11}\left(1-k_{2}\right)} .
\end{aligned}
$$

(ii) $P_{6}$ is a source if

$$
\begin{aligned}
& h>\max \left\{\frac{2}{a_{1}\left(1-k_{1}\right)}, \frac{2}{a_{3}}\right\}, \\
& 0<h<\frac{2 \alpha_{11}}{a_{1} \alpha_{21}\left(1-k_{1}\right)-a_{2} \alpha_{11}\left(1-k_{2}\right)} .
\end{aligned}
$$

(iii) $P_{6}$ is a saddle if

$$
\begin{aligned}
& 0<h<\min \left\{\frac{2}{a_{1}\left(1-k_{1}\right)}, \frac{2}{a_{3}}\right\}, \\
& 0<h<\frac{2 \alpha_{11}}{a_{1} \alpha_{21}\left(1-k_{1}\right)-a_{2} \alpha_{11}\left(1-k_{2}\right)} .
\end{aligned}
$$

(iv) $P_{6}$ is nonhyperbolic if

$$
h=\frac{2}{a_{1}\left(1-k_{1}\right)}
$$

or

$$
h=\frac{2}{a_{3}}
$$

or

$$
h=\frac{2 \alpha_{11}}{a_{1} \alpha_{21}\left(1-k_{1}\right)-a_{2} \alpha_{11}\left(1-k_{2}\right)} .
$$

4.7. Dynamical Behavior about $P_{7}$. From (25), eigenvalues of $\mathrm{JP}_{P_{7}}$ about $P_{7}$ are

$$
\begin{aligned}
& \lambda_{1}=1-h a_{1}\left(1-k_{1}\right) \\
& \lambda_{2}=1-h\left(a_{2}\left(1-k_{2}\right)-\frac{2 a_{1} \alpha_{21}\left(1-k_{1}\right)}{\alpha_{11}}\right) \\
& \lambda_{3}=1+h\left(a_{3}-\frac{a_{2}\left(1-k_{2}\right) \alpha_{32}}{\alpha_{22}}+\frac{a_{1} \alpha_{21} \alpha_{32}\left(1-k_{1}\right)}{\alpha_{11} \alpha_{22}}\right) .
\end{aligned}
$$

The dynamical behavior about $P_{7}$ is concluded as follows:

\section{Lemma 8.}

(i) $P_{7}$ is a sink if

$$
0<h<\min \left\{\frac{2}{a_{1}\left(1-k_{1}\right)}, \frac{2 \alpha_{11}}{a_{2} \alpha_{11}\left(1-k_{2}\right)-2 a_{1} \alpha_{21}\left(1-k_{1}\right)}\right\} \text {, }
$$

$$
h>\frac{2 \alpha_{11} \alpha_{22}}{a_{3} \alpha_{11} \alpha_{22}-a_{2} \alpha_{11} \alpha_{32}\left(1-k_{2}\right)+a_{1} \alpha_{21} \alpha_{32}\left(1-k_{1}\right)} \text {. }
$$

(ii) $P_{7}$ is a source if

$$
\begin{aligned}
& h>\max \left\{\frac{2}{a_{1}\left(1-k_{1}\right)}, \frac{2 \alpha_{11}}{a_{2} \alpha_{11}\left(1-k_{2}\right)-2 a_{1} \alpha_{21}\left(1-k_{1}\right)}\right\}, \\
& 0<h<\frac{2 \alpha_{11} \alpha_{22}}{a_{3} \alpha_{11} \alpha_{22}-a_{2} \alpha_{11} \alpha_{32}\left(1-k_{2}\right)+a_{1} \alpha_{21} \alpha_{32}\left(1-k_{1}\right)} .
\end{aligned}
$$

(iii) $P_{7}$ is a saddle if

$$
\begin{aligned}
& 0<h<\min \left\{\frac{2}{a_{1}\left(1-k_{1}\right)}, \frac{2 \alpha_{11}}{a_{2} \alpha_{11}\left(1-k_{2}\right)-2 a_{1} \alpha_{21}\left(1-k_{1}\right)}\right\}, \\
& 0<h<\frac{2 \alpha_{11} \alpha_{22}}{a_{3} \alpha_{11} \alpha_{22}-a_{2} \alpha_{11} \alpha_{32}\left(1-k_{2}\right)+a_{1} \alpha_{21} \alpha_{32}\left(1-k_{1}\right)} .
\end{aligned}
$$

(iv) $P_{7}$ is nonhyperbolic if

$$
h=\frac{2}{a_{1}\left(1-k_{1}\right)}
$$

or

$$
h=\frac{2 \alpha_{11}}{a_{2} \alpha_{11}\left(1-k_{2}\right)-2 a_{1} \alpha_{21}\left(1-k_{1}\right)},
$$

or

$$
h=\frac{2 \alpha_{11} \alpha_{22}}{a_{3} \alpha_{11} \alpha_{22}-a_{2} \alpha_{11} \alpha_{32}\left(1-k_{2}\right)+a_{1} \alpha_{21} \alpha_{32}\left(1-k_{1}\right)} .
$$

4.8. Dynamical Behavior about $P_{8}$. From (25), eigenvalues of $\left.J\right|_{P_{8}}$ about $P_{8}$ are

$$
\begin{aligned}
& \lambda_{1}=1-h a_{1}\left(1-k_{1}\right), \\
& \lambda_{2}=1-h\left(a_{2}\left(1-k_{2}\right)-\frac{2 a_{1} \alpha_{21}\left(1-k_{1}\right)}{\alpha_{11}}\right), \\
& \lambda_{3}=1-h\left(a_{3}-\frac{a_{2}\left(1-k_{2}\right) \alpha_{32}}{\alpha_{22}}+\frac{a_{1} \alpha_{21} \alpha_{32}\left(1-k_{1}\right)}{\alpha_{11} \alpha_{22}}\right) .
\end{aligned}
$$

The dynamical behavior about $P_{8}$ is concluded as follows. 
Lemma 9.

(i) $P_{8}$ is a sink if

$$
! 0<h<\min \left\{\frac{2}{a_{1}\left(1-k_{1}\right)}, \frac{2 \alpha_{11}}{a_{2} \alpha_{11}\left(1-k_{2}\right)-2 a_{1} \alpha_{21}\left(1-k_{1}\right)}, \frac{2 \alpha_{11} \alpha_{22}}{a_{3} \alpha_{11} \alpha_{22}-a_{2} \alpha_{11} \alpha_{32}\left(1-k_{2}\right)+a_{1} \alpha_{21} \alpha_{32}\left(1-k_{1}\right)}\right\}
$$

(ii) $P_{8}$ is a source if

$$
! h>\max \left\{\frac{2}{a_{1}\left(1-k_{1}\right)}, \frac{2 \alpha_{11}}{a_{2} \alpha_{11}\left(1-k_{2}\right)-2 a_{1} \alpha_{21}\left(1-k_{1}\right)}, \frac{2 \alpha_{11} \alpha_{22}}{a_{3} \alpha_{11} \alpha_{22}-a_{2} \alpha_{11} \alpha_{32}\left(1-k_{2}\right)+a_{1} \alpha_{21} \alpha_{32}\left(1-k_{1}\right)}\right\} .
$$

(iii) $P_{8}$ is a saddle if

$$
\begin{aligned}
& 0<h<\min \left\{\frac{2}{a_{1}\left(1-k_{1}\right)}, \frac{2 \alpha_{11}}{a_{2} \alpha_{11}\left(1-k_{2}\right)-2 a_{1} \alpha_{21}\left(1-k_{1}\right)}\right\}, \\
& h>\frac{2 \alpha_{11} \alpha_{22}}{a_{3} \alpha_{11} \alpha_{22}-a_{2} \alpha_{11} \alpha_{32}\left(1-k_{2}\right)+a_{1} \alpha_{21} \alpha_{32}\left(1-k_{1}\right)} .
\end{aligned}
$$

(iv) $P_{8}$ is nonhyperbolic if

$$
h=\frac{2}{a_{1}\left(1-k_{1}\right)}
$$

or

$$
h=\frac{2 \alpha_{11}}{a_{2} \alpha_{11}\left(1-k_{2}\right)-2 a_{1} \alpha_{21}\left(1-k_{1}\right)},
$$

or

$$
h=\frac{2 \alpha_{11} \alpha_{22}}{a_{3} \alpha_{11} \alpha_{22}-a_{2} \alpha_{11} \alpha_{32}\left(1-k_{2}\right)+a_{1} \alpha_{21} \alpha_{32}\left(1-k_{1}\right)} \text {. }
$$

\section{Periodic Points}

We will prove that $P_{i}(i=1, \ldots, 8)$ of model (15) are periodic points of period $n$.

Theorem 1. Equilibrium points $P_{i}(i=1, \ldots, 8)$ of model (15) are periodic points of prime period 1.

Proof. From (15), define

$$
F(x, y, z):=\left(f_{1}, f_{2}, f_{3}\right),
$$

where $f_{1}, f_{2}$, and $f_{3}$ are represented in (23). From (78), the computation yields

$$
\begin{array}{r}
\left.F\right|_{P_{1}=(0,0,0)}=P_{1}, \\
\left.F\right|_{P_{2}=\left(0,0,\left(a_{3} / \alpha_{33}\right)\right)}=P_{2}, \\
\left.F\right|_{P_{3}=\left(0,\left(1-k_{2}\right) a_{2} / \alpha_{22}, 0\right)}=P_{3}, \\
\left.F\right|_{P_{4}=\left(\left(1-k_{1}\right) a_{1} / \alpha_{11}, 0,0\right)}=P_{4}, \\
\left.F\right|_{P_{5}=\left(0,\left(1-k_{2}\right) a_{2} / \alpha_{22},\left(a_{3} \alpha_{22}-\alpha_{32}\left(1-k_{2}\right) a_{2}\right) / \alpha_{22} \alpha_{33}\right)}=P_{5}, \\
\left.F\right|_{P_{6}=\left(\left(1-k_{1}\right) a_{1} / \alpha_{11}, 0, a_{3} / \alpha_{33}\right)}=P_{6}, \\
\left.F\right|_{P_{7}=\left(\left(1-k_{1}\right) a_{1} / \alpha_{11},\left(a_{2}\left(1-k_{2}\right) \alpha_{11}-a_{1}\left(1-k_{1}\right) \alpha_{21}\right) / \alpha_{11} \alpha_{22}, 0\right)}=P_{7}, \\
\left.F\right|_{P_{8}=\left(\left(1-k_{1}\right) a_{1} / \alpha_{11},\left(a_{2}\left(1-k_{2}\right) \alpha_{11}-a_{1}\left(1-k_{1}\right) \alpha_{21}\right) / \alpha_{11} \alpha_{22},\left(a_{3} \alpha_{11} \alpha_{22}-a_{2}\left(1-k_{2}\right) \alpha_{11} \alpha_{32}+a_{1}\left(1-k_{1}\right) \alpha_{21} \alpha_{32}\right) / \alpha_{11} \alpha_{22} \alpha_{33}\right)}=P_{8} .
\end{array}
$$


Hence, from (79), we can say that equilibrium points $P_{i}(i=1, \ldots, 8)$ of three species model (15) are periodic points of prime period 1 .

Now, it is proved that equilibrium points $P_{i}(i=1, \ldots, 8)$ are period points of period $n$.

Theorem 2. $P_{1}$ of model (15) is a periodic point of period $n$.

Proof. From (78), the following computation yields the required statement:

$$
\begin{aligned}
F^{2}= & \left(f_{1}+h\left[a_{1}\left(1-k_{1}\right) f_{1}-\alpha_{11}\left(f_{1}\right)^{2}\right],\right. \\
& f_{2}+h\left[a_{2}\left(1-k_{2}\right) f_{2}-\alpha_{22}\left(f_{2}\right)^{2}-\alpha_{21} f_{1} f_{2}\right], \\
& \left.f_{3}+h\left[a_{3} f_{3}-\alpha_{33}\left(f_{2}\right)^{2}-\alpha_{32} f_{2} f_{3}\right]\right)\left.\Rightarrow F^{2}\right|_{P_{1}}=P_{1}, \\
F^{3}= & \left(f_{1}^{2}+h\left[a_{1}\left(1-k_{1}\right) f_{1}^{2}-\alpha_{11}\left(f_{1}^{2}\right)^{2}\right],\right. \\
& f_{2}^{2}+h\left[a_{2}\left(1-k_{2}\right) f_{2}^{2}-\alpha_{22}\left(f_{2}^{2}\right)^{2}-\alpha_{21} f_{1}^{2} f_{2}^{2}(x, y, z)\right], \\
& \left.f_{3}^{2}+h\left[a_{3} f_{3}^{2}-\alpha_{33}\left(f_{2}^{2}\right)^{2}-\alpha_{32} f_{2}^{2} f_{3}^{2}\right]\right)\left.\Rightarrow F^{3}\right|_{P_{1}}=P_{1}, \\
\vdots & \\
F^{n}= & \left(f_{1}^{n}+h\left[a_{1}\left(1-k_{1}\right) f_{1}^{n}-\alpha_{11}\left(f_{1}^{n}\right)^{2}\right],\right. \\
& f_{2}^{n}+h\left[a_{2}\left(1-k_{2}\right) f_{2}^{n}-\alpha_{22}\left(f_{2}^{n}\right)^{2}-\alpha_{21} f_{1}^{n} f_{2}^{n}\right], \\
& \left.f_{3}^{n}+h\left[a_{3} f_{3}^{n}-\alpha_{33}\left(f_{2}^{n}\right)^{2}-\alpha_{32} f_{2}^{n} f_{3}^{n}\right]\right)\left.\Rightarrow F^{n}\right|_{P_{1}}=P_{1} .
\end{aligned}
$$

Theorem 3. $P_{2}$ of model (15) is a periodic point of period $n$.

Proof. Utilizing the computation as we have done in (80), one gets the following required statement:

$$
\begin{gathered}
\left.F^{2}\right|_{P_{2}=\left(0,0, a_{3} / \alpha_{33}\right)}=P_{2}, \\
\left.F^{3}\right|_{P_{2}=\left(0,0, a_{3} / \alpha_{33}\right)}=P_{2}, \\
\vdots \\
\left.F^{n}\right|_{P_{2}=\left(0,0, a_{3} / \alpha_{33}\right)}=P_{2} .
\end{gathered}
$$

Theorem 4. $P_{3}$ of model (15) is a periodic point of period $n$.

Proof. In view of (80), one gets the following required statement:

$$
\begin{gathered}
\left.F^{2}\right|_{P_{3}=\left(0,\left(1-k_{2}\right) a_{2} / \alpha_{22}, 0\right)}=P_{3}, \\
\left.F^{3}\right|_{P_{3}=\left(0,\left(1-k_{2}\right) a_{2} / \alpha_{22}, 0\right)}=P_{3}, \\
\vdots \\
\left.F^{n}\right|_{P_{3}=\left(0,\left(1-k_{2}\right) a_{2} / \alpha_{22}, 0\right)}=P_{3} .
\end{gathered}
$$

Theorem 5. $P_{4}$ of model (15) is a periodic point of period $n$.

Proof. In view of (80), one gets the following required statement:

$$
\begin{gathered}
\left.F^{2}\right|_{P_{4}=\left(\left(1-k_{1}\right) a_{1} / \alpha_{11}, 0,0\right)}=P_{4}, \\
\left.F^{3}\right|_{P_{4}=\left(\left(1-k_{1}\right) a_{1} / \alpha_{11}, 0,0\right)}=P_{4}, \\
\vdots \\
\left.F^{n}\right|_{P_{4}=\left(\left(1-k_{1}\right) a_{1} / \alpha_{11}, 0,0\right)}=P_{4} .
\end{gathered}
$$

Theorem 6. $P_{5}$ of model (15) is a periodic point of period $n$.

Proof. From (80), one obtains

$$
\begin{gathered}
\left.F^{2}\right|_{P_{5}=\left(0,\left(1-k_{2}\right) a_{2} / \alpha_{22},\left(a_{3} \alpha_{22}-\alpha_{32}\left(1-k_{2}\right) a_{2}\right) / \alpha_{22} \alpha_{33}\right)}=P_{5}, \\
\left.F^{3}\right|_{P_{5}=\left(0,\left(1-k_{2}\right) a_{2} / \alpha_{22},\left(a_{3} \alpha_{22}-\alpha_{32}\left(1-k_{2}\right) a_{2}\right) / \alpha_{22} \alpha_{33}\right)}=P_{5}, \\
\vdots \\
\left.F^{n}\right|_{P_{5}=\left(0,\left(1-k_{2}\right) a_{2} / \alpha_{22},\left(a_{3} \alpha_{22}-\alpha_{32}\left(1-k_{2}\right) a_{2}\right) / \alpha_{22} \alpha_{33}\right)}=P_{5} .
\end{gathered}
$$

Theorem 7. $P_{6}$ of model (15) is a periodic point of period $n$.

Proof. From (80), one obtains

$$
\begin{aligned}
\left.F^{2}\right|_{P_{6}=\left(\left(1-k_{1}\right) a_{1} / \alpha_{11}, 0, a_{3} / \alpha_{33}\right)}=P_{6}, \\
\left.F^{3}\right|_{P_{6}=\left(\left(1-k_{1}\right) a_{1} / \alpha_{11}, 0, a_{3} / \alpha_{33}\right)}=P_{6}, \\
\vdots \\
\left.F^{n}\right|_{P_{6}=\left(\left(1-k_{1}\right) a_{1} / \alpha_{11}, 0, a_{3} / \alpha_{33}\right)}=P_{6} .
\end{aligned}
$$

Theorem 8. $P_{7}$ of model (15) is a periodic point of period $n$.

Proof. From (80), one obtains

$$
\begin{gathered}
\left.F^{2}\right|_{P_{7}=\left(\left(1-k_{1}\right) a_{1} / \alpha_{11},\left(a_{2}\left(1-k_{2}\right) \alpha_{11}-a_{1}\left(1-k_{1}\right) \alpha_{21}\right) / \alpha_{11} \alpha_{22}, 0\right)}=P_{7}, \\
\left.F^{3}\right|_{P_{7}=\left(\left(1-k_{1}\right) a_{1} / \alpha_{11},\left(a_{2}\left(1-k_{2}\right) \alpha_{11}-a_{1}\left(1-k_{1}\right) \alpha_{21}\right) / \alpha_{11} \alpha_{22}, 0\right)}=P_{7}, \\
\vdots \\
\left.F^{n}\right|_{P_{7}=\left(\left(1-k_{1}\right) a_{1} / \alpha_{11},\left(a_{2}\left(1-k_{2}\right) \alpha_{11}-a_{1}\left(1-k_{1}\right) \alpha_{21}\right) / \alpha_{11} \alpha_{22}, 0\right)}=P_{7} .
\end{gathered}
$$

Theorem 9. $P_{8}$ of model (15) is a periodic point of period $n$.

Proof. From (80), one obtains 


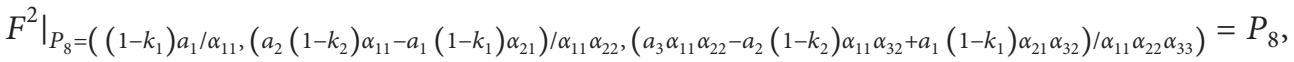

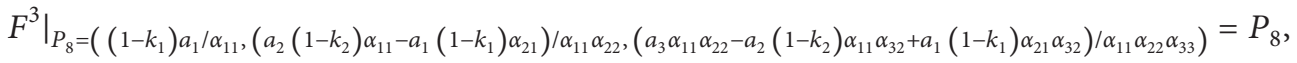

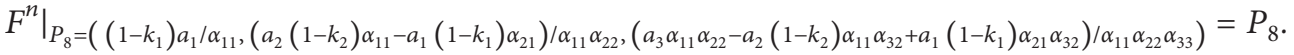

\section{Analysis of Bifurcation}

In this section, we give analysis of bifurcation about fixed points $P_{i}(i=1, \ldots, 8)$ of model (15) by bifurcation theory $[18,19]$.

6.1. Analysis of Bifurcation at $P_{1}$. Here, we will study analysis of bifurcation at $P_{1}$ of model (15). From (26), the simple computation yields $\left.\lambda_{1}\right|_{(29)}=-1$, but $\left.\lambda_{2,3}\right|_{(29)}=1-\left(2 a_{2}(1-\right.$ $\left.\left.k_{2}\right) / a_{1}\left(1-k_{1}\right)\right), 1-\left(2 a_{3} / a_{1}\left(1-k_{1}\right)\right) \neq 1$ or -1 . This suggests that model (15) could undergo a flip bifurcation around $P_{1}$ if $\Omega=\left(h, a_{1}, a_{2}, a_{3}, k_{1}, k_{2}, \alpha_{11}, \alpha_{22}, \alpha_{21}, \alpha_{32}, \alpha_{33}\right)$ passes the curve:

$$
\left.\mathscr{F}\right|_{P_{1}}=\left\{\Omega: h=\frac{2}{a_{1}\left(k_{1}-1\right)}\right\} .
$$

However, flip bifurcation cannot occur by computation, so $P_{1}$ is degenerated with high co-dimension as $\left.\Omega \in \mathscr{F}\right|_{P_{1}}$.

6.2. Analysis of Bifurcation at $P_{2}$. We will study analysis of bifurcation at $P_{2}$ of model (15). From (26), the simple computation yields $\left.\lambda_{3}\right|_{(35)}=-1$, but $\left.\lambda_{1,2}\right|_{(35)}=1+\left(2 a_{1}(1-\right.$ $\left.\left.k_{1}\right) / a_{3}\right), 1+\left(2 a_{2}\left(1-k_{2}\right) / a_{3}\right) \neq 1$ or -1 . This suggests that model (15) could undergo a flip bifurcation around $P_{2}$ if $\Omega$ passes the curve:

$$
\left.\mathscr{F}\right|_{P_{2}}=\left\{\Omega: h=\frac{2}{a_{3}}\right\} .
$$

The proof of following theorem shows that model (15) undergoes flip bifurcation around $P_{2}$ if $\left.\Omega \in \mathscr{F}\right|_{P_{2}}$.

Theorem 10. Model (15) undergo flip bifurcation around $P_{2}$ if $\left.\Omega \in \mathscr{F}\right|_{P_{2}}$.

Proof. It is noticed that three-species model (15) is invariant with respect to $x=y=0$. Thus, we restrict (15) on $x=y=0$, to determine the bifurcation, where it takes the form

$$
z_{n+1}=z_{n}+h\left(a_{3} z_{n}-\alpha_{33} z_{n}^{2}\right)
$$

From (90), define

$$
f(z):=z+h\left(a_{3} z-\alpha_{33} z^{2}\right) .
$$

Now, one denotes $h=h^{*}=\left(2 / a_{3}\right)$ and $z=z^{*}=$ $\left(a_{3} / \alpha_{33}\right)$. The computation yields

$$
\begin{gathered}
\left.f_{z}\right|_{h=h^{*}=\left(2 / a_{3}\right), z=z^{*}=\left(a_{3} / \alpha_{33}\right)}=-1, \\
\left.f_{z z}\right|_{h=h^{*}=\left(2 / a_{3}\right), z=z^{*}=\left(a_{3} / \alpha_{33}\right)}=-\frac{4 \alpha_{33}}{a_{3}} \neq 0, \\
\left.f_{h}\right|_{h=h^{*}=\left(2 / a_{3}\right), z=z^{*}=\left(a_{3} / \alpha_{33}\right)}=-\frac{a_{3}^{2}}{\alpha_{33}} \neq 0 .
\end{gathered}
$$

From (92)-(94), it can be concluded that the model undergoes flip bifurcation around $P_{2}$ if $\left.\Omega \in \mathscr{F}\right|_{P_{2}}$.

6.3. Analysis of Bifurcation at $P_{3}$. From (38), the computation yields $\left.\lambda_{2}\right|_{(42)}=-1$, but $\left.\lambda_{1,3}\right|_{(42)}=1+\left(2 a_{1}(1-\right.$ $\left.\left.k_{1}\right) / a_{2}\left(1-k_{2}\right)\right), 1+\left(2 / a_{2}\left(1-k_{2}\right)\right)\left[a_{3} \alpha_{22}-a_{2} \alpha_{32}\left(1-k_{2}\right)\right]$ $\neq 1$ or -1 . This suggests that model (15) could undergo a flip bifurcation around $P_{3}$ if $\Omega$ passes the curve:

$$
\left.\mathscr{F}\right|_{P_{3}}=\left\{\Omega: h=\frac{2}{a_{2}\left(1-k_{2}\right)}\right\} .
$$

The proof of following theorem shows that model (15) undergoes flip bifurcation around $P_{3}$ if $\left.\Omega \in \mathscr{F}\right|_{P_{3}}$.

Theorem 11. Model (15) undergoes flip bifurcation around $P_{3}$ if $\left.\Omega \in \mathscr{F}\right|_{P_{3}}$

Proof. It is noticed that, w.r.t $x=z=0$, model (15) is invariant. So, one restricts model (15) on $x=z=0$, where it becomes

$$
y_{n+1}=y_{n}+h\left(a_{2}\left(1-k_{2}\right) y_{n}-\alpha_{22} y_{n}^{2}\right)
$$

From (96), define

$$
f(y):=y+h a_{2}\left(1-k_{2}\right) y-h \alpha_{22} y^{2} .
$$

Denote $h=h^{*}=\left(2 / a_{2}\left(1-k_{2}\right)\right)$ and $y=y^{*}=\left(a_{2}(1-\right.$ $\left.\left.k_{2}\right) / \alpha_{22}\right)$. By computation, one obtains 


$$
\begin{aligned}
& \left.f_{y}\right|_{h=h^{*}=\left(2 / a_{2}\left(1-k_{2}\right)\right), y=y^{*}=\left(a_{2}\left(1-k_{2}\right) / \alpha_{22}\right)}=-1, \\
& \left.f_{y y}\right|_{h=h^{*}=\left(2 / a_{2}\left(1-k_{2}\right)\right), y=y^{*}=\left(a_{2}\left(1-k_{2}\right) / \alpha_{22}\right)}=-\frac{4 \alpha_{22}}{a_{2}\left(1-k_{2}\right)} \neq 0, \\
& \left.f_{h}\right|_{h=h^{*}=\left(2 / a_{2}\left(1-k_{2}\right)\right), y=y^{*}=\left(a_{2}\left(1-k_{2}\right) / \alpha_{22}\right)}=\frac{a_{2}\left(1-k_{2}\right)}{\alpha_{22}} \neq 0 .
\end{aligned}
$$

So, model (15) undergoes flip bifurcation by (98)-(100) if $\left.\Omega \in \mathscr{F}\right|_{P_{3}}$.

6.4. Analysis of Bifurcation at $P_{4}$. From (45), the computation yields $\left.\lambda_{1}\right|_{(48)}=-1$, but $\left.\lambda_{2,3}\right|_{(48)}=1+\left(2 a_{2}\left(1-k_{2}\right) /\right.$ $\left.a_{1}\left(1-k_{1}\right)\right), 1+\left(2 a_{3} / a_{1}\left(1-k_{1}\right)\right) \neq 1$ or -1 . This suggests that model (15) could undergo a flip bifurcation around $P_{4}$ if $\Omega$ passes the curve:

$$
\left.\mathscr{F}\right|_{P_{3}}=\left\{\Omega: h=\frac{2}{a_{1}\left(1-k_{1}\right)}\right\} .
$$

The proof of the following theorem shows that model (15) undergoes flip bifurcation around $P_{4}$ if $\left.\Omega \in \mathscr{F}\right|_{P_{4}}$.

Theorem 12. Model (15) undergoes flip bifurcation around $P_{4}$ if $\left.\Omega \in \mathscr{F}\right|_{P_{4}}$.

Proof. It is noticed that, w.r.t $y=z=0$, model (15) is invariant. So, one restricts model (15) on $y=z=0$, where it becomes

$$
x_{n+1}=x_{n}+h\left(a_{1}\left(1-k_{1}\right) x_{n}-\alpha_{11} x_{n}^{2}\right) .
$$

From (102), define

$$
f(x):=x+h a_{1}\left(1-k_{1}\right) x-h \alpha_{11} x^{2} .
$$

Denote $\quad h=h^{*}=\left(2 / a_{1}\left(1-k_{1}\right)\right), x=x^{*}=\left(a_{1}(1-\right.$ $\left.\left.k_{1}\right) / \alpha_{11}\right)$. By computation, one obtains

$$
\begin{gathered}
\left.f_{x}\right|_{h=h^{*}=\left(2 / a_{1}\left(1-k_{1}\right)\right), x=x^{*}=\left(a_{1}\left(1-k_{1}\right) / \alpha_{11}\right)=-1,} \\
\left.f_{x x}\right|_{h=h^{*}=\left(2 / a_{1}\left(1-k_{1}\right)\right), x=x^{*}=\left(a_{1}\left(1-k_{1}\right) / \alpha_{11}\right)}=-\frac{4 \alpha_{11}}{a_{1}\left(1-k_{1}\right)} \neq 0, \\
\left.f_{h}\right|_{h=h^{*}=\left(2 / a_{1}\left(1-k_{1}\right)\right), x=x^{*}=\left(a_{1}\left(1-k_{1}\right) / \alpha_{11}\right)}=\frac{a_{1}\left(1-k_{1}\right)}{\alpha_{11}} \neq 0 .
\end{gathered}
$$

So, model (15) undergoes flip bifurcation by (104)-(106) if $\left.\Omega \in \mathscr{F}\right|_{P_{4}}$.

6.5. Analysis of Bifurcation at $P_{5}$. From (50), the computation yields $\lambda_{1} l_{(54)}=-1$, but $\left.\lambda_{2,3}\right|_{(54)}=1+\left(2 a_{2}(1-\right.$ $\left.\left.k_{2}\right) / a_{1}\left(1-k_{1}\right)\right), 1-\left(2 / a_{1}\left(1-k_{1}\right)\right)\left[-a_{3}+\left(\alpha_{32}\left(1-k_{2}\right) a_{2} /\right.\right.$ $\left.\alpha_{22}\right)$ ] $\neq 1$ or -1 . This suggests that model (15) could undergo flip bifurcation around $P_{5}$ if $\Omega$ passes the curve:

$$
\left.\mathscr{F}\right|_{P_{5}}=\left\{\Omega: h=\frac{2}{a_{1}\left(k_{1}-1\right)}\right\} .
$$

The proof of the following theorem shows that model (15) undergoes flip bifurcation around $P_{5}$ if $\left.\Omega \in \mathscr{F}\right|_{P_{5}}$.

Theorem 13. Model (15) undergoes flip bifurcation around $P_{5}$ if $\left.\Omega \in \mathscr{F}\right|_{P_{5}}$

Proof. Recall that if $\left.\Omega \in \mathscr{F}\right|_{P_{5}}$, then $\left.\lambda_{1}\right|_{(54)}=-1$, but $\left.\lambda_{2,3}\right|_{(54)}=1+\left(2 a_{2}\left(1-k_{2}\right) / a_{1}\left(1-k_{1}\right)\right), 1-\left(2 / a_{1}\left(1-k_{1}\right)\right)$ $\left[-a_{3}+\left(\alpha_{32}\left(1-k_{2}\right) a_{2} / \alpha_{22}\right)\right] \neq 1$ or -1 . So, hereafter, detailed flip bifurcation is explored if $\Omega$ varies in the nbhd of $h$, i.e., $h=h+\varepsilon$, by assuming $h \neq\left(2 \alpha_{22} /\left(a_{3} \alpha_{22}-a_{2} \alpha_{32}(1-\right.\right.$ $\left.\left.\left.k_{2}\right)\right)\right), 2 / a_{2}\left(1-k_{2}\right)$. Let

$$
\begin{aligned}
& u_{n}=x_{n}, \\
& v_{n}=y_{n}-\frac{\left(1-k_{2}\right) a_{2}}{\alpha_{22}}, \\
& w_{n}=z_{n}-\frac{a_{3} \alpha_{22}-\alpha_{32}\left(1-k_{2}\right) a_{2}}{\alpha_{22} \alpha_{33}} .
\end{aligned}
$$


Then, (15) gives

$$
\begin{aligned}
& \left(\begin{array}{c}
u_{n+1} \\
v_{n+1} \\
w_{n+1}
\end{array}\right)=\left(\begin{array}{ccc}
1+h a_{1}\left(1-k_{1}\right) & 0 & 0 \\
-h \alpha_{21} \frac{\left(1-k_{2}\right)}{\alpha_{22}} a_{2} & 1-h a_{2}\left(1-k_{2}\right) & 0 \\
0 & -h \alpha_{32}\left(\frac{a_{3} \alpha_{32}-\alpha_{32}\left(1-k_{2}\right) a_{2}}{\alpha_{22} \alpha_{33}}\right) & 1+h\left(-a_{3}+\frac{\alpha_{32}\left(1-k_{2}\right) a_{2}}{\alpha_{22}}\right)
\end{array}\right)\left(\begin{array}{c}
u_{n} \\
v_{n} \\
w_{n}
\end{array}\right) \\
& +\left(\begin{array}{c}
h \alpha_{11} u_{n}^{2}+a_{1}\left(1-k_{1}\right) \varepsilon u_{n}-\alpha_{11} \varepsilon u_{n}^{2} \\
h\left[\frac{\left(a_{2}\left(1-k_{2}\right)\right)^{2}}{\alpha_{22}}-\alpha_{22}\left(v_{n}+\frac{\left(1-k_{2}\right) a_{2}}{\alpha_{22}}\right)^{2}+\alpha_{21} u_{n} v_{n}\right]+ \\
\varepsilon\left[a_{2}\left(1-k_{2}\right)\left(v_{n}+\frac{a_{2}\left(1-k_{2}\right)}{\alpha_{22}}\right)-\alpha_{22}\left(v_{n}+\frac{\left(1-k_{2}\right) a_{2}}{\alpha_{22}}\right)^{2}-\alpha_{21} u_{n}\left(v_{n}+\frac{a_{2}\left(1-k_{2}\right)}{\alpha_{22}}\right)\right] \\
h\left[a_{3}\left(\frac{a_{3} \alpha_{32}-\alpha_{32}\left(1-k_{2}\right) a_{2}}{\alpha_{22} \alpha_{33}}\right)-\alpha_{33}\left(w_{n}+\frac{a_{3} \alpha_{22}-\alpha_{32}\left(1-k_{2}\right) a_{2}}{\alpha_{22} \alpha_{33}}\right)^{2}-\right. \\
\left.\alpha_{32} v_{n} w_{n}-\frac{\left(1-k_{2}\right) a_{2}}{\alpha_{22}}\left(\frac{a_{3} \alpha_{32}-\alpha_{32}\left(1-k_{2}\right) a_{2}}{\alpha_{22} \alpha_{33}}\right)\right]+\varepsilon\left[a_{3}\left(w_{n}+\frac{a_{3} \alpha_{32}-\alpha_{32}\left(1-k_{2}\right) a_{2}}{\alpha_{22} \alpha_{33}}\right)-\right. \\
\left.\alpha_{33}\left(w_{n}+\frac{a_{3} \alpha_{22}-\alpha_{32}\left(1-k_{2}\right) a_{2}}{\alpha_{22} \alpha_{33}}\right)^{2}-\alpha_{32}\left(v_{n}+\frac{\left(1-k_{2}\right) a_{2}}{\alpha_{22}}\right)\left(w_{n}+\frac{a_{3} \alpha_{22}-\alpha_{32}\left(1-k_{2}\right) a_{2}}{\alpha_{22} \alpha_{33}}\right)\right]
\end{array}\right) .
\end{aligned}
$$

By using transformation,

$$
\left(\begin{array}{c}
u_{n} \\
v_{n} \\
w_{n}
\end{array}\right)=\left(\begin{array}{ccc}
a_{11} & 0 & 0 \\
a_{21} & a_{22} & 0 \\
1 & 1 & 1
\end{array}\right)\left(\begin{array}{l}
x_{n} \\
y_{n} \\
z_{n}
\end{array}\right)
$$

(109) takes the form

$$
\left(\begin{array}{c}
x_{n+1} \\
y_{n+1} \\
z_{n+1}
\end{array}\right)=\left(\begin{array}{ccc}
-1 & 0 & 0 \\
0 & \lambda_{2} & 0 \\
0 & 0 & \lambda_{3}
\end{array}\right)\left(\begin{array}{l}
x_{n} \\
y_{n} \\
z_{n}
\end{array}\right)+\left(\begin{array}{l}
F\left(x_{n}, y_{n}, z_{n}, \varepsilon\right) \\
G\left(x_{n}, y_{n}, z_{n}, \varepsilon\right) \\
H\left(x_{n}, y_{n}, z_{n}, \varepsilon\right)
\end{array}\right),
$$


where

$$
\begin{aligned}
& a_{11}=\frac{\left(-a_{1}-a_{2}+a_{1} k_{1}+a_{2} k_{2}\right) \alpha_{22}\left(a_{1} \alpha_{22}+a_{3} \alpha_{22}-a_{1} k_{1} \alpha_{22}-a_{2} \alpha_{32}+a_{2} k_{2} \alpha_{32}\right) \alpha_{33}}{a_{2}\left(-1+k_{2}\right)\left(-a_{2}+a_{3}+a_{2} k_{2}\right) \alpha_{21} \alpha_{32}^{2}}, \\
& a_{21}=-\frac{\left(a_{1} \alpha_{22}+a_{3} \alpha_{22}-a_{1} k_{1} \alpha_{22}-a_{2} \alpha_{32}+a_{2} k_{2} \alpha_{32}\right) \alpha_{33}}{\left(-a_{2}+a_{3}+a_{2} k_{2}\right) \alpha_{32}^{2}}, \\
& a_{22}=-\frac{\left(-a_{2} \alpha_{22}+a_{3} \alpha_{22}+a_{2} k_{2} \alpha_{22}-a_{2} \alpha_{32}+a_{2} k_{2} \alpha_{32}\right) \alpha_{33}}{\left(-a_{2}+a_{3}+a_{2} k_{2}\right) \alpha_{32}^{2}}, \\
& F=x_{n} a_{1}\left(1-k_{1}\right) \varepsilon-x_{n}^{2} a_{11} \alpha_{11}+h x_{n}^{2} a_{11} \alpha_{11}-x_{n}^{2} a_{11} \alpha_{11} \varepsilon, \\
& G=-\frac{a_{1} a_{11}\left(1-k_{1}\right) \varepsilon-x_{n}^{2} a_{11}^{2} \alpha_{11}+h x_{n}^{2} a_{11}^{2} \alpha_{11}-x_{n}^{2} \varepsilon a_{11}^{2} \alpha_{11}}{a_{11}} \\
& +\frac{h\left(\left(a_{2}^{2}\left(1-k_{2}\right)^{2} / \alpha_{22}\right)+x_{n} a_{11} \alpha_{21}\left(x_{n} a_{21}+y_{n} \alpha_{22}\right)-\alpha_{22}\left(x_{n} a_{21}+y_{n} \alpha_{22} 1-k_{2} / \alpha_{22}\right)^{2}\right)}{a_{21}} \\
& +\frac{\varepsilon a_{2}\left(1-k_{2}\right)\left(x_{n} a_{21}+y_{n} \alpha_{22}+\left(a_{2}\left(1-k_{2}\right) / \alpha_{22}\right)\right)-x_{n} \varepsilon a_{11} \alpha_{21}\left(x_{n} a_{21}+y_{n} \alpha_{22}+\left(a_{2}\left(1-k_{2}\right) / \alpha_{22}\right)\right)}{a_{21}} \\
& -\frac{\varepsilon \alpha_{22}\left(x_{n} a_{21}+y_{n} \alpha_{22}+\left(a_{2}\left(1-k_{2}\right) / \alpha_{11}\right)\right)^{2}}{a_{21}}, \\
& H=h\left[-x_{n}\left(x_{n}+y_{n}+z_{n}\right) a_{11} \alpha_{32}-\frac{a_{2}\left(1-k_{2}\right) a_{3} \alpha_{32}-a_{2} \alpha_{32}\left(1-k_{2}\right)}{\alpha_{22}^{2} \alpha_{33}}\right. \\
& \left.+\frac{a_{3}\left(a_{3} \alpha_{32}-a_{2} \alpha_{32}\left(1-k_{2}\right)\right)}{\alpha_{22} \alpha_{33}}-\left(x_{n}+y_{n}+z_{n}+\frac{a_{3} \alpha_{32}-a_{2} \alpha_{32}\left(1-k_{2}\right)}{\alpha_{22} \alpha_{33}}\right)^{2} \alpha_{33}\right] \\
& -\frac{h\left(\left(a_{2}^{2}\left(1-k_{2}\right)^{2} / \alpha_{22}\right)+x_{n} a_{11} \alpha_{21}\left(x_{n} a_{21}+y_{n} \alpha_{22}\right)-\alpha_{22}\left(x_{n} a_{21}+y_{n} \alpha_{22}\left(\left(1-k_{2}\right) / \alpha_{22}\right)\right)^{2}\right)}{a_{21}} \\
& +\frac{\varepsilon a_{2}\left(1-k_{2}\right)\left(x_{n} a_{21}+y_{n} \alpha_{22}+\left(a_{2}\left(1-k_{2}\right) / \alpha_{22}\right)\right)-x_{n} \varepsilon a_{11} \alpha_{21}\left(x_{n} a_{21}+y_{n} \alpha_{22}+\left(a_{2}\left(1-k_{2}\right) / \alpha_{22}\right)\right)}{a_{21}} \\
& -\frac{\varepsilon \alpha_{22}\left(x_{n} a_{21}+y_{n} \alpha_{22}+\left(a_{2}\left(1-k_{2}\right) / \alpha_{11}\right)\right)^{2}}{a_{21}} \\
& \varepsilon a_{3}\left(x_{n}+y_{n}+z_{n}+\frac{a_{3} \alpha_{32}-a_{2} \alpha_{32}\left(1-k_{2}\right)}{\alpha_{22} \alpha_{33}}\right) \\
& -\varepsilon\left(x_{n} a_{21}+y_{n} \alpha_{22}+\frac{a_{2}\left(1-k_{2}\right)}{\alpha_{22}}\right) \alpha_{32}\left(x_{n}+y_{n}+z_{n}+\frac{a_{3} \alpha_{32}-a_{2}\left(1-k_{2}\right) \alpha_{32}}{\alpha_{22} \alpha_{33}}\right) \\
& -\left(x_{n}+y_{n}+z_{n}+\frac{a_{3} \alpha_{32}-a_{2} \alpha_{32}\left(1-k_{2}\right)}{\alpha_{22} \alpha_{33}}\right)^{2} \alpha_{33} .
\end{aligned}
$$


Now, consider (111) on the center manifold, i.e.,

$$
W^{c}(0)=\left\{\left(x_{n}, y_{n}, z_{n}\right) \mid\left(y_{n}, z_{n}\right)=\left(\chi_{1}\left(x_{n}\right), \chi_{2}\left(x_{n}\right)\right), \chi_{i}(0)=0, D \chi_{i}(0)=0, i=1,2\right\},
$$

where

$$
\chi_{i}\left(x_{n}\right)=a_{i} x^{2}+b_{i} x^{3}+O(x)^{4}, \quad \text { for } i=1,2 .
$$

From (111) and (113), one obtains

$$
\begin{aligned}
& \chi_{1}\left(-x_{n}+F\left(x_{n}, \chi_{1}, \chi_{2}\right)\right)=\lambda_{2} \chi_{1}\left(x_{n}\right)+G\left(x_{n}, \chi_{1}, \chi_{2}\right), \\
& \chi_{2}\left(-x_{n}+F\left(x_{n}, \chi_{1}, \chi_{2}\right)\right)=\lambda_{3} \chi_{2}\left(x_{n}\right)+H\left(x_{n}, \chi_{1}, \chi_{2}\right) .
\end{aligned}
$$

From (115), computation yields $a_{1}=b_{1}=a_{2}=b_{2}=0$. Finally, map (111); restrict to $W^{c}(0)$ as

$$
f\left(x_{n}\right)=-x_{n}+x_{n} a_{1}\left(1-k_{1}\right) \varepsilon-x_{n}^{2} a_{11} \alpha_{11}+h x_{n}^{2} a_{11} \alpha_{11}-x_{n}^{2} a_{11} \alpha_{11} \varepsilon+O\left(\left(\left|x_{n}\right|+|\varepsilon|\right)^{3}\right) .
$$

For the model to undergo flip bifurcation, the following should be nonzero:

$$
\begin{aligned}
& \Omega_{1}=\left.\left(\frac{\partial^{2} f}{\partial x_{n} \partial \varepsilon}+\frac{1}{2} \frac{\partial f}{\partial \varepsilon} \frac{\partial^{2} f}{\partial x_{n}^{2}}\right)\right|_{O}=a_{1}\left(1-k_{1}\right) \neq 0, \\
& \Omega_{2}=\left.\left(\frac{1}{6} \frac{\partial^{3} f}{\partial x_{n}^{3}}+\left(\frac{1}{2} \frac{\partial^{2} f}{\partial x_{n}^{2}}\right)^{2}\right)\right|_{O}=\left(a_{11} \alpha_{11}-h a_{11} \alpha_{11}\right)^{2}>0 .
\end{aligned}
$$

From (117), one can say that about $P_{5}$ model (15) undergoes flip bifurcation if $\left.\Omega \in \mathscr{F}\right|_{P_{5}}$. Moreover, period-2 points bifurcating from $P_{5}$ are stable since $\Omega_{2}=\left(a_{11} \alpha_{11}-h a_{11} \alpha_{11}\right)^{2}>0$.

6.6. Analysis of Bifurcation at $P_{6}$. From (57), the computation yields $\left.\lambda_{1}\right|_{(61)}=-1$, but $\left.\lambda_{2,3}\right|_{(61)}=1+\left(2 / a_{1}\left(1-k_{1}\right)\right)$ $\left[a_{2}\left(1-k_{2}\right)-\left(a_{1} \alpha_{21}\left(1-k_{1}\right) / \alpha_{11}\right)\right], 1-\left(2 a_{3} / a_{1}\left(1-k_{1}\right)\right)$ $\neq 1$ or -1 . This suggests that model (15) could undergo flip bifurcation around $P_{6}$ if $\Omega$ passes the curve:

$$
\left.\mathscr{F}\right|_{P_{6}}=\left\{\Omega: h=\frac{2}{a_{1}\left(1-k_{1}\right)}\right\} .
$$

The proof of the following theorem shows that model (15) undergoes flip bifurcation around $P_{6}$ if $\left.\Omega \in \mathscr{F}\right|_{P_{6}}$.

Theorem 14. Model (15) undergoes flip bifurcation around $P_{6}$ if $\left.\Omega \in \mathscr{F}\right|_{P_{6}}$.

Proof. Recall that if $\left.\Omega \in \mathscr{F}\right|_{P_{6}}$, then $\left.\lambda_{1}\right|_{(61)}=-1$, but $\left.\lambda_{2,3}\right|_{(61)}=1+\left(2 / a_{1}\left(1-k_{1}\right)\right)\left[a_{2}\left(1-k_{2}\right)-\left(a_{1} \alpha_{21}\left(1-k_{1}\right) /\right.\right.$ $\left.\left.\alpha_{11}\right)\right], 1-\left(2 a_{3} / a_{1}\left(1-k_{1}\right)\right) \neq 1$ or -1 . So, hereafter, detailed flip bifurcation is explored if $\Omega$ varies in the nbhd of $h$, i.e., $h=h+\varepsilon$, by assuming $h \neq\left(2 / a_{3}\right), 2 \alpha_{11} /\left(a_{1} \alpha_{21}\left(1-k_{1}\right)-\right.$ $\left.a_{2} \alpha_{11}\left(1-k_{2}\right)\right)$. Let

$$
\begin{aligned}
& u_{n}=x_{n}-\frac{\left(1-k_{1}\right) a_{1}}{\alpha_{11}}, \\
& v_{n}=y_{n}, \\
& w_{n}=z_{n}-\frac{a_{3}}{\alpha_{33}} .
\end{aligned}
$$

Then, (15) gives 


$$
\begin{aligned}
\left(\begin{array}{c}
u_{n+1} \\
v_{n+1} \\
v_{n+1}
\end{array}\right)= & \left(\begin{array}{ccc}
1-h a_{1}\left(1-k_{1}\right) & 0 \\
0 & 1+h\left(a_{2}\left(1-k_{2}\right)-\frac{a_{1} \alpha_{21}\left(1-k_{1}\right)}{\alpha_{11}}\right) & 0
\end{array}\right)\left(\begin{array}{c}
u_{n} \\
v_{n} \\
w_{n}
\end{array}\right) \\
& +\left(h \frac{\left(a_{1}\left(1-k_{1}\right)\right)^{2}}{\alpha_{11}}-h \alpha_{11}\left(u_{n}+\frac{a_{1}\left(1-k_{1}\right)}{\alpha_{11}}\right)^{2}+\varepsilon a_{1}\left(1-k_{1}\right)\left(u_{n}+\frac{a_{1}\left(1-k_{1}\right)}{\alpha_{11}}\right)-\varepsilon \alpha_{11}\left(u_{n}+\frac{a_{1}\left(1-k_{1}\right)}{\alpha_{11}}\right)^{2}\right. \\
& -h \alpha_{22} v_{n}^{2}-h \alpha_{22} u_{n} v_{n}+\varepsilon a_{2}\left(1-k_{2}\right) v_{n}-\varepsilon \alpha_{22} v_{n}^{2}-\varepsilon \alpha_{21} v_{n}\left(u_{n}+\frac{a_{1}\left(1-k_{1}\right)}{\alpha_{11}}\right) \\
& h\left(\frac{a_{3}^{2}}{\alpha_{33}}-\alpha_{33}\left(w_{n}+\frac{a_{3}}{\alpha_{33}}\right)^{2}-\alpha_{32} v_{n} w_{n}\right)^{2} \\
& \left.+\varepsilon a_{3}\left(w_{n}+\frac{a_{3}}{\alpha_{33}}\right)-\varepsilon \alpha_{33}\left(w_{n}+\frac{a_{3}}{\alpha_{33}}\right)^{2}-\varepsilon \alpha_{32} v_{n}\left(w_{n}+\frac{a_{3}}{\alpha_{33}}\right)\right)
\end{aligned}
$$

Using transformation,

$$
\left(\begin{array}{c}
u_{n} \\
v_{n} \\
w_{n}
\end{array}\right)=\left(\begin{array}{ccc}
0 & 1 & 0 \\
0 & 0 & b_{23} \\
1 & 0 & 1
\end{array}\right)\left(\begin{array}{c}
x_{n} \\
y_{n} \\
z_{n}
\end{array}\right)
$$

$$
\left(\begin{array}{c}
x_{n+1} \\
y_{n+1} \\
z_{n+1}
\end{array}\right)=\left(\begin{array}{ccc}
-1 & 0 & 0 \\
0 & \lambda_{2} & 0 \\
0 & 0 & \lambda_{3}
\end{array}\right)\left(\begin{array}{l}
x_{n} \\
y_{n} \\
z_{n}
\end{array}\right)+\left(\begin{array}{l}
F_{1}\left(x_{n}, y_{n}, z_{n}, \varepsilon\right) \\
G_{1}\left(x_{n}, y_{n}, z_{n}, \varepsilon\right) \\
H_{1}\left(x_{n}, y_{n}, z_{n}, \varepsilon\right)
\end{array}\right),
$$

(120) becomes

$$
\begin{aligned}
b_{23}= & \frac{\alpha_{33}\left(-a_{2} \alpha_{11}-a_{3} \alpha_{11}+a_{2} k_{2} \alpha_{11}+a_{1} \alpha_{21}-a_{1} k_{1} \alpha_{21}\right)}{a_{3} \alpha_{11} \alpha_{32}}, \\
F_{1}= & -z_{n} a_{2}\left(1-k_{2}\right) \varepsilon+z_{n}\left(y_{n}+\frac{a_{1}\left(1-k_{1}\right)}{\alpha_{11}}\right) \alpha_{21} \varepsilon+h z_{n} \alpha_{22}+h y_{n} z_{n} \alpha_{22}+z_{n}^{2} b_{23} \alpha_{22} \varepsilon \\
& +a_{3}\left(x_{n}+y_{n}+\frac{a_{3}}{\alpha_{33}}\right) \varepsilon-z_{n} b_{23} \alpha_{32}\left(x_{n}+y_{n}+\frac{a_{3}}{\alpha_{33}}\right) \varepsilon-\left(x_{n}+y_{n}+\frac{a_{3}}{\alpha_{33}}\right)^{2} \alpha_{33} \varepsilon \\
& +h\left(-\left(x_{n}+y_{n}\right) z_{n} b_{23} \alpha_{32}+\frac{a_{3}^{2}}{\alpha_{33}}-\left(x_{n}+y_{n}+\frac{a_{3}}{\alpha_{33}}\right)^{2} \alpha_{33}\right), \\
G_{1}= & a_{1}\left(1-k_{1}\right)\left(y_{n}+\frac{a_{1}\left(1-k_{1}\right)}{\alpha_{11}}\right) \varepsilon-h\left(y_{n}+\frac{a_{1}\left(1-k_{1}\right)}{\alpha_{11}}\right)^{2} \\
& +\frac{h a_{1}^{2}\left(1-k_{1}\right)^{2}}{\alpha_{11}}-\left(y_{n}+\frac{a_{1}\left(1-k_{1}\right)}{\alpha_{11}}\right)^{2} \alpha_{11} \varepsilon, \\
H_{1}= & -z_{n} a_{2}\left(1-k_{2}\right) \varepsilon+z_{n}\left(y_{n}+\frac{a_{1}\left(1-k_{1}\right)}{\alpha_{11}}\right) \alpha_{21} \varepsilon+h z_{n} \alpha_{22}+h y_{n} z_{n} \alpha_{22}+z_{n}^{2} b_{23} \alpha_{22} \varepsilon .
\end{aligned}
$$


Now, from model (122) on the center manifold,

$$
W^{c}(0)=\left\{\left(x_{n}, y_{n}, z_{n}\right) \mid\left(y_{n}, z_{n}\right)=\left(\chi_{3}\left(x_{n}\right), \chi_{4}\left(x_{n}\right)\right), \chi_{i}(0)=0, D \chi_{i}(0)=0, i=3,4\right\},
$$

where

$$
\chi_{i}\left(x_{n}\right)=a_{i} x^{2}+b_{i} x^{3}+O(x)^{4}, \quad \text { for } i=3,4 .
$$

From (122) and (124), one has

$$
\begin{aligned}
& \chi_{3}\left(-x_{n}+F_{1}\left(x_{n}, \chi_{3}, \chi_{4}\right)\right)=\lambda_{2} \chi_{3}\left(x_{n}\right)+G_{1}\left(x_{n}, \chi_{3}, \chi_{4}\right), \\
& \chi_{4}\left(-x_{n}+F_{1}\left(x_{n}, \chi_{3}, \chi_{4}\right)\right)=\lambda_{3} \chi_{4}\left(x_{n}\right)+H_{1}\left(x_{n}, \chi_{3}, \chi_{4}\right) .
\end{aligned}
$$

From (126), the calculation yields: $a_{3}=b_{3}=a_{4}=b_{4}=0$. Thus, map (122); restrict to $W^{c}(0)$ as

$$
f\left(x_{n}\right)=-x_{n}+a_{3}\left(x_{n}+\frac{a_{3}}{\alpha_{33}}\right) \varepsilon-\left(x_{n}+\frac{a_{3}}{\alpha_{33}}\right)^{2} \alpha_{33} \varepsilon+h\left(\frac{a_{3}^{2}}{\alpha_{33}}-\left(x_{n}+\frac{a_{3}}{\alpha_{33}}\right)^{2} \alpha_{33}\right) .
$$

From (117) and (127), the computation yields: $\Omega_{1}=3 a_{3}-\left(h a_{3}^{2} / \alpha_{33}\right) \neq 0$ and $\Omega_{2}=h^{2} \alpha_{33}^{2}>0$. This implies that about $P_{6}$ model (15) undergoes flip bifurcation if $\left.\Omega \in \mathscr{F}\right|_{P_{6}}$. Moreover, period-2 points bifurcating from $P_{6}$ are stable since $\Omega_{2}=h^{2} \alpha_{33}^{2}>0$.

6.7. Analysis of Bifurcation at $P_{7}$. From (64), the computation yields $\left.\lambda_{1}\right|_{(68)}=-1$, but $\left.\lambda_{2,3}\right|_{(68)}=1-\left(2 / a_{1}\left(1-k_{1}\right)\right)$ $\left[a_{2}\left(1-k_{2}\right)-\left(2 a_{1} \alpha_{21}\left(1-k_{1}\right) / \alpha_{11}\right)\right], 1+\left(2 / a_{1}\left(1-k_{1}\right)\right)\left[a_{3}-\right.$ $\left.\left(a_{2}\left(1-k_{2}\right) \alpha_{32} / \alpha_{22}\right)+\left(a_{1} \alpha_{21} \alpha_{32}\left(1-k_{1}\right) / \alpha_{11} \alpha_{22}\right)\right] \neq 1$ or -1 . This suggests that model (15) could undergo flip bifurcation around $P_{7}$ if $\Omega$ passes the curve:

$$
\left.\mathscr{F}\right|_{P_{7}}=\left\{\Omega: h=\frac{2}{a_{1}\left(1-k_{1}\right)}\right\} \text {. }
$$

The proof of the following theorem shows that model (15) undergoes flip bifurcation around $P_{7}$ if $\left.\Omega \in \mathscr{F}\right|_{P_{7}}$.
Theorem 15. Model (15) undergoes flip bifurcation around $P_{7}$ if $\left.\Omega \in \mathscr{F}\right|_{P_{7}}$

Proof. Recall that if $\left.\Omega \in \mathscr{F}\right|_{P_{7}}$, then $\left.\lambda_{1}\right|_{(68)}=-1$, but $\left.\lambda_{2,3}\right|_{(68)}=1-\left(2 / a_{1}\left(1-k_{1}\right)\right)\left[a_{2}\left(1-k_{2}\right)-\left(2 a_{1} \alpha_{21}\left(1-k_{1}\right) /\right.\right.$ $\left.\left.\alpha_{11}\right)\right], 1+\left(2 / a_{1}\left(1-k_{1}\right)\right)\left[a_{3}-\left(a_{2}\left(1-k_{2}\right) \alpha_{32} / \alpha_{22}\right)+\left(a_{1} \alpha_{21}\right.\right.$ $\left.\left.\alpha_{32}\left(1-k_{1}\right) / \alpha_{11} \alpha_{22}\right)\right] \neq 1$ or -1 . So, in the following, flip bifurcation is explored by assuming $h \neq\left(2 \alpha_{11} /\left(a_{2} \alpha_{11}\left(1-k_{2}\right)-\right.\right.$ $\left.\left.2 a_{1} \alpha_{21}\left(1-k_{1}\right)\right)\right),\left(2 \alpha_{11} \alpha_{22} / a_{3} \alpha_{11} \alpha_{22}-a_{2} \alpha_{11} \alpha_{32}\left(1-k_{2}\right)+\right.$ $\left.a_{1} \alpha_{21} \alpha_{32}\left(1-k_{1}\right)\right)$. Let

$$
\begin{aligned}
& u_{n}=x_{n}-\frac{\left(1-k_{1}\right) a_{1}}{\alpha_{11}}, \\
& v_{n}=y_{n}-\frac{a_{2}\left(1-k_{2}\right) \alpha_{11}-a_{1}\left(1-k_{1}\right) \alpha_{21}}{\alpha_{11} \alpha_{22}}, \\
& w_{n}=z_{n} .
\end{aligned}
$$




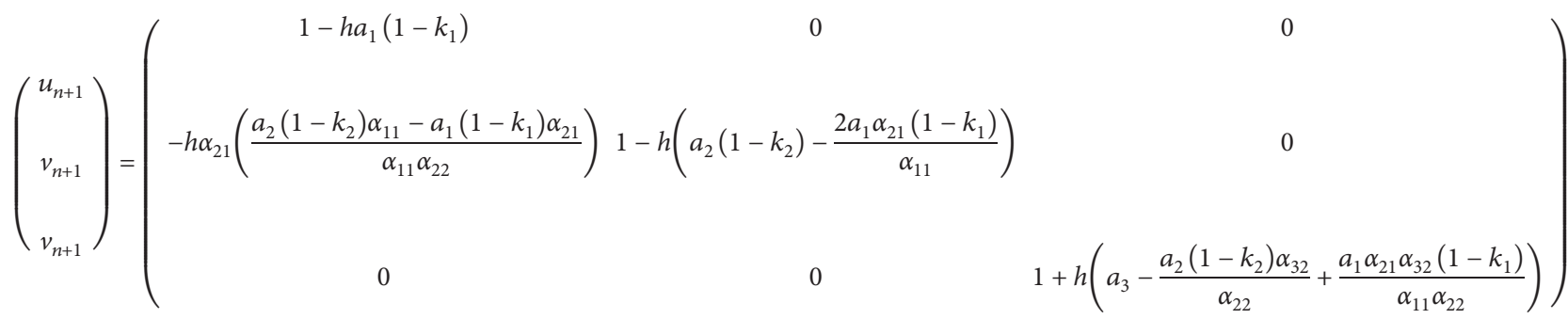

$$
\begin{aligned}
& \left(\begin{array}{c}
u_{n} \\
v_{n} \\
w_{n}
\end{array}\right) \\
& +\left(h\left(\frac{\left(a_{1}\left(1-k_{1}\right)\right)^{2}}{\alpha_{11}}-\alpha_{11}\left(u_{n}+\frac{a_{1}\left(1-k_{1}\right)}{\alpha_{11}}\right)^{2}\right)\right. \\
& +\varepsilon\left(a_{1}\left(1-k_{1}\right)\left(u_{n}+\frac{a_{1}\left(1-k_{1}\right)}{\alpha_{11}}\right)-\alpha_{11}\left(u_{n}+\frac{a_{1}\left(1-k_{1}\right)}{\alpha_{11}}\right)^{2}\right) \\
& \cdot h\left[a_{2}\left(1-k_{2}\right)\left(\frac{a_{2}\left(1-k_{2}\right) \alpha_{11}-a_{1}\left(1-k_{1}\right) \alpha_{21}}{\alpha_{11} \alpha_{22}}\right)+\alpha_{22}\left(v_{n}+\frac{a_{2}\left(1-k_{2}\right) \alpha_{11}-a_{1}\left(1-k_{1}\right) \alpha_{21}}{\alpha_{11} \alpha_{22}}\right)^{2}\right. \\
& \left.-\alpha_{21} \frac{a_{1}\left(1-k_{1}\right)}{\alpha_{11}}\left(\frac{a_{2}\left(1-k_{2}\right) \alpha_{11}-a_{1}\left(1-k_{1}\right) \alpha_{21}}{\alpha_{11} \alpha_{22}}\right)\right]+\varepsilon\left[a_{2}\left(1-k_{2}\right)\left(v_{n}+\frac{a_{2}\left(1-k_{2}\right) \alpha_{11}-a_{1}\left(1-k_{1}\right) \alpha_{21}}{\alpha_{11} \alpha_{22}}\right)\right. \\
& -\alpha_{22}\left(v_{n}+\frac{a_{2}\left(1-k_{2}\right) \alpha_{11}-a_{1}\left(1-k_{1}\right) \alpha_{21}}{\alpha_{11} \alpha_{22}}\right)^{2} \\
& \left.-\alpha_{21}\left(u_{n}+\frac{a_{1}\left(1-k_{1}\right)}{\alpha_{11}}\right)\left(v_{n}+\frac{a_{2}\left(1-k_{2}\right) \alpha_{11}-a_{1}\left(1-k_{1}\right) \alpha_{21}}{\alpha_{11} \alpha_{22}}\right)\right] h\left(-\alpha_{33} w_{n}^{2}-\alpha_{32} v_{n} w_{n}\right)+\varepsilon\left(a_{3} w_{n}-\alpha_{33} w_{n}^{2}\right. \\
& \left.\left.-\alpha_{32} w_{n}\left(v_{n}+\frac{a_{2}\left(1-k_{2}\right) \alpha_{11}-a_{1}\left(1-k_{1}\right) \alpha_{21}}{\alpha_{11} \alpha_{22}}\right)\right)\right) .
\end{aligned}
$$

Using transformation,

$$
\left(\begin{array}{c}
u_{n} \\
v_{n} \\
w_{n}
\end{array}\right)=\left(\begin{array}{ccc}
c_{11} & 0 & 0 \\
1 & 1 & 0 \\
0 & 0 & 1
\end{array}\right)\left(\begin{array}{c}
x_{n} \\
y_{n} \\
z_{n}
\end{array}\right)
$$

\section{(130) gives}

$$
\left(\begin{array}{c}
x_{n+1} \\
y_{n+1} \\
z_{n+1}
\end{array}\right)=\left(\begin{array}{ccc}
-1 & 0 & 0 \\
0 & \lambda_{2} & 0 \\
0 & 0 & \lambda_{3}
\end{array}\right)\left(\begin{array}{c}
x_{n} \\
y_{n} \\
z_{n}
\end{array}\right)+\left(\begin{array}{l}
F_{2}\left(x_{n}, y_{n}, z_{n}, \varepsilon\right) \\
G_{2}\left(x_{n}, y_{n}, z_{n}, \varepsilon\right) \\
H_{2}\left(x_{n}, y_{n}, z_{n}, \varepsilon\right)
\end{array}\right),
$$


where

$$
\begin{aligned}
c_{11}= & \frac{\alpha_{11} \alpha_{22}\left(h a_{1}\left(1-k_{1}\right)-h a_{2}\left(1-k_{2}\right)-\left(2 h a_{1}\left(1-k_{1}\right) \alpha_{21} / \alpha_{11}\right)\right)}{h \alpha_{21}\left(a_{2}\left(1-k_{2}\right) \alpha_{11}-a_{1}\left(1-k_{1}\right) \alpha_{21}\right)}, \\
F_{2}= & \frac{h\left[\left(\left(a_{1}\left(1-k_{1}\right)\right)^{2} / \alpha_{11}\right)-\left(x_{n} c_{11}+\left(a_{1}\left(1-k_{1}\right) / \alpha_{11}\right)\right)^{2} \alpha_{11}\right]+\varepsilon\left[a_{1}\left(1-k_{1}\right)\left(x_{n} c_{11}+\left(a_{1}\left(1-k_{1}\right) / \alpha_{11}\right)\right)-\left(x_{n} c_{11}+\left(a_{1}\left(1-k_{1}\right) / \alpha_{11}\right)\right)^{2} \alpha_{11}\right]}{c_{11}}, \\
G_{2}= & h\left[\frac{a_{2}\left(1-k_{2}\right)\left(a_{2} a_{11}\left(1-k_{2}\right)-a_{1}\left(1-k_{1}\right) \alpha_{21}\right)}{\alpha_{11} \alpha_{22}}-\frac{a_{1}\left(1-k_{1}\right) \alpha_{21}\left(a_{2} a_{11}\left(1-k_{2}\right)-a_{1}\left(1-k_{1}\right) \alpha_{21}\right)}{\alpha_{11}^{2} \alpha_{22}}\right. \\
& +\left(x_{n}+y_{n}+\frac{\left.\left.a_{2} a_{11}\left(1-k_{2}\right)-a_{1}\left(1-k_{1}\right) \alpha_{21}\right)^{2} \alpha_{22}\right]}{\alpha_{11} \alpha_{22}}\right. \\
& . \frac{h\left[\left(\left(a_{1}\left(1-k_{1}\right)\right)^{2} / \alpha_{11}\right)-\left(x_{n} c_{11}+\left(a_{1}\left(1-k_{1}\right) / \alpha_{11}\right)\right)^{2} \alpha_{11}\right]+\varepsilon\left[a_{1}\left(1-k_{1}\right)\left(x_{n} c_{11}+\left(a_{1}\left(1-k_{1}\right) / \alpha_{11}\right)\right)-\left(x_{n} c_{11}+\left(a_{1}\left(1-k_{1}\right) / \alpha_{11}\right)\right)^{2} \alpha_{11}\right]}{c_{11}} \\
& +\varepsilon\left[a_{2}\left(1-k_{2}\right)\left(x_{n}+y_{n}+\frac{a_{2} a_{11}\left(1-k_{2}\right)-a_{1}\left(1-k_{1}\right) \alpha_{21}}{\alpha_{11} \alpha_{22}}\right)\right. \\
& \left.+\frac{a_{1}\left(1-k_{1}\right) \alpha_{21}\left(x_{n}+y_{n}+\left(\left(a_{2} a_{11}\left(1-k_{2}\right)-a_{1}\left(1-k_{1}\right) \alpha_{21}\right) / \alpha_{11} \alpha_{22}\right)\right)^{3}\left(x_{n} c_{11}\left(\left(a_{2} a_{11}\left(1-k_{2}\right)-a_{1}\left(1-k_{1}\right) \alpha_{21}\right) / \alpha_{11} \alpha_{22}\right)\right) \alpha_{22}}{a_{11}}\right], \\
H_{2}= & h\left(-\left(x_{n}+y_{n}\right) z_{n} \alpha_{32}-z^{2} \alpha_{33}\right)+\varepsilon\left[z_{n} a_{3}-z_{n}\left(x_{n}+y_{n}+\frac{\left.\left.a_{2} a_{11}\left(1-k_{2}\right)-a_{1}\left(1-k_{1}\right) \alpha_{21}\right) \alpha_{32}-z_{n}^{2} \alpha_{33}\right] .}{\alpha_{11} \alpha_{22}}\right.\right.
\end{aligned}
$$

Now, using system (132) on the center manifold,

$$
W^{c}(0)=\left\{\left(x_{n}, y_{n}, z_{n}\right) \mid\left(y_{n}, z_{n}\right)=\left(\chi_{5}\left(x_{n}\right), \chi_{6}\left(x_{n}\right)\right), \chi_{i}(0)=0, D \chi_{i}(0)=0, i=5,6\right\},
$$

where

$$
\chi_{i}\left(x_{n}\right)=a_{i} x^{2}+b_{i} x^{3}+O(x)^{4}, \quad \text { for } i=5,6
$$

In view of (132) and (134), we obtain

$$
\begin{aligned}
& \chi_{5}\left(-x_{n}+F_{2}\left(x_{n}, \chi_{5}, \chi_{6}\right)\right)=\lambda_{2} \chi_{5}\left(x_{n}\right)+G_{2}\left(x_{n}, \chi_{5}, \chi_{6}\right), \\
& \chi_{6}\left(-x_{n}+F_{2}\left(x_{n}, \chi_{5}, \chi_{6}\right)\right)=\lambda_{3} \chi_{6}\left(x_{n}\right)+H_{2}\left(x_{n}, \chi_{5}, \chi_{6}\right) .
\end{aligned}
$$


From (136), one gets: $a_{5}=b_{5}=a_{6}=b_{6}=0$. Finally, map (132); restrict to $W^{c}(0)$ as

$$
f\left(x_{n}\right)=-x_{n}+\frac{h\left[\left(\left(a_{1}\left(1-k_{1}\right)\right)^{2} / \alpha_{11}\right)-\left(x_{n} c_{11}+\left(a_{1}\left(1-k_{1}\right) / \alpha_{11}\right)\right)^{2} \alpha_{11}\right]+\varepsilon\left[a_{1}\left(1-k_{1}\right)\left(x_{n} c_{11}+\left(a_{1}\left(1-k_{1}\right) / \alpha_{11}\right)\right)-\left(x_{n} c_{11}+\left(a_{1}\left(1-k_{1}\right) / \alpha_{11}\right)\right)^{2} \alpha_{11}\right]}{c_{11}} .
$$

From (117) and (137), the computation yields: $\Omega_{1}=a_{1}(1$ $\left.-k_{1}\right)\left(1-2 c_{11}\right) \neq 0$. Moreover, $\Omega_{2}=\left(h \alpha_{11} c_{11}+2 \alpha_{11} c_{11} \varepsilon\right)^{2}$ $>0$. This implies that about $P_{7}$ model (15) undergoes flip bifurcation if $\left.\Omega \in \mathscr{F}\right|_{P_{7}}$. Moreover, period-2 points bifurcating from $P_{7}$ are stable since $\Omega_{2}=\left(h \alpha_{11} c_{11}+\right.$ $\left.2 \alpha_{11} c_{11} \varepsilon\right)^{2}>0$.

6.8. Analysis of Bifurcation at $P_{8}$. From (71), the computation yields $\left.\lambda_{1}\right|_{(75)}=-1$, but $\left.\lambda_{2,3}\right|_{(75)}=1-\left(2 / a_{1}\left(1-k_{1}\right)\right)$ $\left[a_{2}\left(1-k_{2}\right)-\left(2 a_{1} \alpha_{21}\left(1-k_{1}\right) / \alpha_{11}\right)\right], 1-\left(2 / a_{1}\left(1-k_{1}\right)\right)\left[a_{3}-\right.$ $\left.\left(a_{2}\left(1-k_{2}\right) \alpha_{32} / \alpha_{22}\right)+\left(a_{1} \alpha_{21} \alpha_{32}\left(1-k_{1}\right) / \alpha_{11} \alpha_{22}\right)\right] \neq 1$ or -1 . This suggests that model (15) could undergo flip bifurcation around $P_{8}$ if $\Omega$ passes the curve:

$$
\left.\mathscr{F}\right|_{P_{8}}=\left\{\Omega: h=\frac{2}{a_{1}\left(1-k_{1}\right)}\right\} \text {. }
$$

The proof of the following theorem shows that model (15) undergoes flip bifurcation around $P_{8}$ if $\left.\Omega \in \mathscr{F}\right|_{P_{8}}$.
Proof. Recall that if $\left.\Omega \in \mathscr{F}\right|_{P_{8}}$, then $\left.\lambda_{1}\right|_{(75)}=-1$, but $\lambda_{2,3}$ $\mathrm{I}_{(75)}=1-\left(2 / a_{1}\left(1-k_{1}\right)\right)\left[a_{2}\left(1-k_{2}\right)-\left(2 a_{1} \alpha_{21}\left(1-k_{1}\right) / \alpha_{11}\right)\right.$ ], $1-\left(2 / a_{1}\left(1-k_{1}\right)\right)\left[a_{3}-\left(a_{2}\left(1-k_{2}\right) \alpha_{32} / \alpha_{22}\right)+\left(a_{1} \alpha_{21} \alpha_{32}\right.\right.$ $\left.\left.\left(1-k_{1}\right) / \alpha_{11} \alpha_{22}\right)\right] \neq 1$ or -1 . So, in the following, flip bifurcation is explored by assuming $h \neq\left(2 \alpha_{11} /\left(a_{2} \alpha_{11}\left(1-k_{2}\right)\right.\right.$ $\left.\left.-2 a_{1} \alpha_{21}\left(1-k_{1}\right)\right)\right),\left(2 \alpha_{11} \alpha_{22} /\left(a_{3} \alpha_{11} \alpha_{22}-a_{2} \alpha_{11} \alpha_{32}\left(1-k_{2}\right)+\right.\right.$ $\left.\left.a_{1} \alpha_{21} \alpha_{32}\left(1-k_{1}\right)\right)\right)$. Let

$$
\begin{aligned}
& u_{n}=x_{n}-\frac{\left(1-k_{1}\right) a_{1}}{\alpha_{11}}, \\
& v_{n}=y_{n}-\frac{a_{2}\left(1-k_{2}\right) \alpha_{11}-a_{1}\left(1-k_{1}\right) \alpha_{21}}{\alpha_{11} \alpha_{22}}, \\
& w_{n}=z_{n}-\frac{a_{3} \alpha_{11} \alpha_{22}-a_{2}\left(1-k_{2}\right) \alpha_{11} \alpha_{32}+a_{1}\left(1-k_{1}\right) \alpha_{21} \alpha_{32}}{\alpha_{11} \alpha_{22} \alpha_{33}} .
\end{aligned}
$$

Then, (15) becomes

Theorem 16. Model (15) undergoes flip bifurcation around $P_{8}$ if $\left.\Omega \in \mathscr{F}\right|_{P_{8}}$.

$$
\begin{aligned}
\left(\begin{array}{c}
u_{n+1} \\
v_{n+1} \\
v_{n+1}
\end{array}\right)= & 0 \\
-h \alpha_{21}\left(\frac{a_{2}\left(1-k_{2}\right) \alpha_{11}-a_{1}\left(1-k_{1}\right) \alpha_{21}}{\alpha_{11} \alpha_{22}}\right. & 1-h\left(a_{2}\left(1-k_{2}\right)-\frac{2 a_{1} \alpha_{21}\left(1-k_{1}\right)}{\alpha_{11}}\right) \\
0 & -h \alpha_{32}\left(\frac{a_{3} \alpha_{11} \alpha_{22}-a_{2}\left(1-k_{2}\right) \alpha_{11} \alpha_{32}+a_{1}\left(1-k_{1}\right) \alpha_{21} \alpha_{32}}{\alpha_{11} \alpha_{22} \alpha_{33}}\right) 1-h\left(a_{3}-\frac{\left.a_{2}\left(1-k_{2}\right) \alpha_{32}+\frac{a_{1} \alpha_{21} \alpha_{32}\left(1-k_{1}\right)}{\alpha_{11} \alpha_{22}}\right)}{\alpha_{22}}\right) \\
& \left(\begin{array}{c}
u_{n} \\
v_{n} \\
w_{n}
\end{array}\right)+\left(\begin{array}{c}
F_{3}\left(x_{n}, y_{n}, z_{n}, \varepsilon\right) \\
G_{3}\left(x_{n}, y_{n}, z_{n}, \varepsilon\right) \\
H_{3}\left(x_{n}, y_{n}, z_{n}, \varepsilon\right)
\end{array}\right),
\end{aligned}
$$


where

$$
\begin{aligned}
& F_{3}=h\left(\frac{\left(a_{1}\left(1-k_{1}\right)\right)^{2}}{\alpha_{11}}-\alpha_{11}\left(u_{n}+\frac{a_{1}\left(1-k_{1}\right)}{\alpha_{11}}\right)^{2}\right)+\varepsilon\left(a_{1}\left(1-k_{1}\right)\left(u_{n}+\frac{a_{1}\left(1-k_{1}\right)}{\alpha_{11}}\right)-\alpha_{11}\left(u_{n}+\frac{a_{1}\left(1-k_{1}\right)}{\alpha_{11}}\right)^{2}\right), \\
& G_{3}=h\left(a_{2}\left(1-k_{2}\right)\left(\frac{a_{2}\left(1-k_{2}\right) \alpha_{11}-a_{1}\left(1-k_{1}\right) \alpha_{21}}{\alpha_{11} \alpha_{22}}\right)\right. \\
& -\alpha_{21}\left(v_{n}+\frac{a_{2}\left(1-k_{2}\right) \alpha_{11}-a_{1}\left(1-k_{1}\right) \alpha_{21}}{\alpha_{11} \alpha_{22}}\right)^{2}-\alpha_{21} u_{n} v_{n} \\
& \left.-\alpha_{21} \frac{a_{1}\left(1-k_{1}\right)}{\alpha_{11}}\left(\frac{a_{2}\left(1-k_{2}\right) \alpha_{11}-a_{1}\left(1-k_{1}\right) \alpha_{21}}{\alpha_{11} \alpha_{22}}\right)\right) \\
& +\varepsilon\left(a_{2}\left(1-k_{2}\right)\left(v_{n}+\frac{a_{2}\left(1-k_{2}\right) \alpha_{11}-a_{1}\left(1-k_{1}\right) \alpha_{21}}{\alpha_{11} \alpha_{22}}\right)-\alpha_{22}\left(v_{n}+\frac{a_{2}\left(1-k_{2}\right) \alpha_{11}-a_{1}\left(1-k_{1}\right) \alpha_{21}}{\alpha_{11} \alpha_{22}}\right)^{2}\right. \\
& \left.-\alpha_{21}\left(u_{n}+\frac{a_{1}\left(1-k_{1}\right)}{\alpha_{11}}\right)\left(v_{n}+\frac{a_{2}\left(1-k_{2}\right) \alpha_{11}-a_{1}\left(1-k_{1}\right) \alpha_{21}}{\alpha_{11} \alpha_{22}}\right)\right) \\
& H_{3}=h\left(a_{3}\left(\frac{a_{3} \alpha_{11} \alpha_{22}-a_{2}\left(1-k_{2}\right) \alpha_{11} \alpha_{32}+a_{1}\left(1-k_{1}\right) \alpha_{21} \alpha_{32}}{\alpha_{11} \alpha_{22} \alpha_{33}}\right)-\alpha_{32} v_{n} w_{n}\right. \\
& -\alpha_{33}\left(w_{n}+\frac{a_{3} \alpha_{11} \alpha_{22}-a_{2}\left(1-k_{2}\right) \alpha_{11} \alpha_{32}+a_{1}\left(1-k_{1}\right) \alpha_{21} \alpha_{32}}{\alpha_{11} \alpha_{22} \alpha_{33}}\right)^{2} \\
& \left.-\alpha_{32} \frac{a_{2}\left(1-k_{2}\right) \alpha_{11}-a_{1}\left(1-k_{1}\right) \alpha_{21}}{\alpha_{11} \alpha_{22}}\left(\frac{a_{3} \alpha_{11} \alpha_{22}-a_{2}\left(1-k_{2}\right) \alpha_{11} \alpha_{32}+a_{1}\left(1-k_{1}\right) \alpha_{21} \alpha_{32}}{\alpha_{11} \alpha_{22} \alpha_{33}}\right)\right) \\
& +\varepsilon\left(a_{3}\left(w_{n}+\frac{a_{3} \alpha_{11} \alpha_{22}-a_{2}\left(1-k_{2}\right) \alpha_{11} \alpha_{32}+a_{1}\left(1-k_{1}\right) \alpha_{21} \alpha_{32}}{\alpha_{11} \alpha_{22} \alpha_{33}}\right)\right. \\
& \left.-\alpha_{33}\left(w_{n}+\frac{a_{3} \alpha_{11} \alpha_{22}-a_{2}\left(1-k_{2}\right) \alpha_{11} \alpha_{32}+a_{1}\left(1-k_{1}\right) \alpha_{21} \alpha_{32}}{\alpha_{11} \alpha_{22} \alpha_{33}}\right)^{2}\right)-\alpha_{32}\left(v_{n}+\frac{a_{2}\left(1-k_{2}\right) \alpha_{11}-a_{1}\left(1-k_{1}\right) \alpha_{21}}{\alpha_{11} \alpha_{22}}\right) \\
& \varepsilon \times\left(w_{n}+\frac{a_{3} \alpha_{11} \alpha_{22}-a_{2}\left(1-k_{2}\right) \alpha_{11} \alpha_{32}+a_{1}\left(1-k_{1}\right) \alpha_{21} \alpha_{32}}{\alpha_{11} \alpha_{22} \alpha_{33}}\right) .
\end{aligned}
$$

Now, by utilizing transformation,

$$
\left(\begin{array}{c}
u_{n} \\
v_{n} \\
w_{n}
\end{array}\right)=\left(\begin{array}{ccc}
d_{11} & 0 & 0 \\
d_{21} & d_{22} & 0 \\
1 & 1 & 1
\end{array}\right)\left(\begin{array}{l}
x_{n} \\
y_{n} \\
z_{n}
\end{array}\right),
$$

gives

$$
\left(\begin{array}{l}
x_{n+1} \\
y_{n+1} \\
z_{n+1}
\end{array}\right)=\left(\begin{array}{ccc}
-1 & 0 & 0 \\
0 & \lambda_{2} & 0 \\
0 & 0 & \lambda_{3}
\end{array}\right)\left(\begin{array}{l}
x_{n} \\
y_{n} \\
z_{n}
\end{array}\right)+\left(\begin{array}{l}
F_{2}^{*}\left(x_{n}, y_{n}, z_{n}, \varepsilon\right) \\
G_{2}^{*}\left(x_{n}, y_{n}, z_{n}, \varepsilon\right) \\
H_{2}^{*}\left(x_{n}, y_{n}, z_{n}, \varepsilon\right)
\end{array}\right),
$$


where

$$
\begin{aligned}
& d_{11}=\frac{\alpha_{11}^{2}\left(h a_{1}-h a_{1} k_{1}-h a_{2}\left(1-k_{2}\right)-\left(2 h a_{1}\left(1-k_{1}\right) \alpha_{21} / \alpha_{11}\right)\right) \alpha_{22}^{2} \alpha_{33}\left(h a_{1}-h a_{1} k_{1}-h\left(a_{3}-\left(a_{2}\left(1-k_{2}\right) \alpha_{32} / \alpha_{22}\right)+\left(a_{1}\left(1-k_{1}\right) \alpha_{21} \alpha_{32} / \alpha_{11} \alpha_{22}\right)\right)\right)}{h^{2} \alpha_{21}\left(a_{2}\left(1-k_{2}\right) \alpha_{11}-a_{1}\left(1-k_{1}\right) \alpha_{21}\right) \alpha_{32}\left(a_{3} \alpha_{11} \alpha_{22}-a_{2}\left(1-k_{2}\right) \alpha_{11} \alpha_{32}+a_{1}\left(1-k_{1}\right) \alpha_{21} \alpha_{32}\right)}, \\
& d_{21}=\frac{\alpha_{11} \alpha_{22}\left(h a_{1}-h a_{1} k_{1}-h\left(a_{3}-\left(a_{2}\left(1-k_{2}\right) \alpha_{32} / \alpha_{22}\right)+\left(a_{1}\left(1-k_{1}\right) \alpha_{21} \alpha_{32} / \alpha_{11} \alpha_{22}\right)\right)\right) \alpha_{33}}{h \alpha_{32}\left(a_{3} \alpha_{11} \alpha_{22}-a_{2}\left(1-k_{2}\right) \alpha_{11} \alpha_{32}+a_{1}\left(1-k_{1}\right) \alpha_{21} \alpha_{32}\right)}, \\
& d_{22}=-\frac{\alpha_{33}\left(-a_{2} \alpha_{11} \alpha_{22}+a_{3} \alpha_{11} \alpha_{22}+a_{2} k_{2} \alpha_{11} \alpha_{22}-2 a_{1} \alpha_{21} \alpha_{22}+2 a_{1} k_{1} \alpha_{21} \alpha_{22}-a_{2} \alpha_{11} \alpha_{32}+a_{2} k_{2} \alpha_{11} \alpha_{32}+a_{1} \alpha_{21} \alpha_{32}-a_{1} k_{1} \alpha_{21} \alpha_{32}\right)}{\alpha_{32}\left(a_{3} \alpha_{11} \alpha_{22}-a_{2} \alpha_{11} \alpha_{32}+a_{2} k_{2} \alpha_{11} \alpha_{32}+a_{1} \alpha_{21} \alpha_{32}-a_{1} k_{1} \alpha_{21} \alpha_{32}\right)}, \\
& F_{2}^{*}=\frac{h\left(\left(\left(a_{1}\left(1-k_{1}\right)\right)^{2} / \alpha_{11}\right)-\left(x_{n} d_{11}+\left(a_{1}\left(1-k_{1}\right) / \alpha_{11}\right)\right)^{2} \alpha_{11}\right)+\varepsilon\left(a_{1}\left(1-k_{1}\right)\left(x_{n} d_{11}+\left(a_{1}\left(1-k_{1}\right) / \alpha_{11}\right)\right)-\left(x_{n} d_{11}+\left(a_{1}\left(1-k_{1}\right) / \alpha_{11}\right)\right)^{2} \alpha_{11}\right)}{d_{11}}, \\
& G_{2}^{*}=-\frac{d_{21}\left(h\left(\left(\left(a_{1}\left(1-k_{1}\right)\right)^{2} / \alpha_{11}\right)-\left(x_{n} d_{11}+\left(a_{1}\left(1-k_{1}\right) / \alpha_{11}\right)\right)^{2} \alpha_{11}\right)+\varepsilon\left(a_{1}\left(1-k_{1}\right)\left(x_{n} d_{11}+\left(a_{1}\left(1-k_{1}\right) / \alpha_{11}\right)\right)-\left(x_{n} d_{11}+\left(a_{1}\left(1-k_{1}\right) / \alpha_{11}\right)\right)^{2} \alpha_{11}\right)\right)}{d_{11} d_{22}} \\
& +\frac{h}{d_{22}}\left(-x_{n} d_{11}\left(d_{21} x_{n}+d_{22} y_{n}\right) \alpha_{21}-\alpha_{21}\left(d_{21} x_{n}+d_{22} y_{n}+\frac{a_{2} a_{11}\left(1-k_{2}\right)-a_{1}\left(1-k_{1}\right) \alpha_{21}}{\alpha_{11} \alpha_{22}}\right)^{2}\right. \\
& \left.+\frac{a_{2}\left(1-k_{2}\right)\left(a_{2} a_{11}\left(1-k_{2}\right)-a_{1}\left(1-k_{1}\right) \alpha_{21}\right)}{\alpha_{11} \alpha_{22}}-\frac{a_{1}\left(1-k_{1}\right) \alpha_{21}\left(a_{2} a_{11}\left(1-k_{2}\right)-a_{1}\left(1-k_{1}\right) \alpha_{21}\right)}{\alpha_{11}^{2} \alpha_{22}}\right) \\
& +\frac{\varepsilon}{d_{22}}\left(a_{2}\left(1-k_{2}\right)\left(d_{21} x_{n}+d_{22} y_{n}+\frac{a_{2} a_{11}\left(1-k_{2}\right)-a_{1}\left(1-k_{1}\right) \alpha_{21}}{\alpha_{11} \alpha_{22}}\right)\right. \\
& -\frac{a_{1}\left(1-k_{1}\right) \alpha_{21}\left(d_{11} x+\left(a_{1}\left(1-k_{1}\right) / \alpha_{11}\right)\right)\left(d_{21} x_{n}+d_{22} y_{n}+\left(\left(a_{2} a_{11}\left(1-k_{2}\right)-a_{1}\left(1-k_{1}\right) \alpha_{21}\right) / \alpha_{11} \alpha_{22}\right)\right)}{\alpha_{11}} \\
& \left.-\left(d_{21} x_{n}+d_{22} y_{n}+\frac{a_{2} a_{11}\left(1-k_{2}\right)-a_{1}\left(1-k_{1}\right) \alpha_{21}}{\alpha_{11} \alpha_{22}}\right)^{2} \alpha_{22}\right) \\
& H_{2}^{*}=h\left(-\left(y_{n}+2 z_{n}\right)\left(d_{21} x_{n}+d_{22} y_{n}\right) \alpha_{32}-\alpha_{32}\left(x_{n}+y_{n}+z_{n}+\frac{a_{3} \alpha_{11} \alpha_{22}-a_{2}\left(1-k_{2}\right) \alpha_{11} \alpha_{32}+a_{1}\left(1-k_{1}\right) \alpha_{21} \alpha_{32}}{\alpha_{11} \alpha_{22} \alpha_{33}}\right)^{2}\right. \\
& +\frac{a_{3}\left(a_{3} \alpha_{11} \alpha_{22}-a_{2}\left(1-k_{2}\right) \alpha_{11} \alpha_{32}+a_{1}\left(1-k_{1}\right) \alpha_{21} \alpha_{32}\right)}{\alpha_{11} \alpha_{22} \alpha_{33}} \\
& \left.-\frac{\left(a_{2} a_{11}\left(1-k_{2}\right)-a_{1}\left(1-k_{1}\right) \alpha_{21}\right) \alpha_{32}\left(a_{3} \alpha_{11} \alpha_{22}-a_{2}\left(1-k_{2}\right) \alpha_{11} \alpha_{32}+a_{1}\left(1-k_{1}\right) \alpha_{21} \alpha_{32}\right)}{\alpha_{11}^{2} \alpha_{22}^{2} \alpha_{33}}\right) \\
& +\frac{\left(d_{21}-d_{22}\right)\left(h\left(\left(\left(a_{1}\left(1-k_{1}\right)\right)^{2} / \alpha_{11}\right)-\left(d_{11} x_{n}+\left(a_{1}\left(1-k_{1}\right) / \alpha_{11}\right)\right)^{2} \alpha_{11}\right)+\epsilon\left(a_{1}\left(1-k_{1}\right)\left(d_{11} x_{n}+\left(a_{1}\left(1-k_{1}\right) / \alpha_{11}\right)\right)-\left(d_{11} x_{n}+\left(a_{1}\left(1-k_{1}\right) / \alpha_{11}\right)\right)^{2} \alpha_{11}\right)\right)}{d_{11} d_{22}} \\
& -\frac{h}{d_{22}}\left(-d_{11} x_{n}\left(d_{21} x_{n}+d_{22} y_{n}\right) \alpha_{21}-\alpha_{21}\left(d_{21} x_{n}+d_{22} y_{n}+\frac{a_{2} a_{11}\left(1-k_{2}\right)-a_{1}\left(1-k_{1}\right) \alpha_{21}}{\alpha_{11} \alpha_{22}}\right)^{2}\right. \\
& \left.+\frac{a_{2}\left(1-k_{2}\right)\left(a_{2} a_{11}\left(1-k_{2}\right)-a_{1}\left(1-k_{1}\right) \alpha_{21}\right)}{\alpha_{11} \alpha_{22}}-\frac{a_{1}\left(1-k_{1}\right) \alpha_{21}\left(a_{2} a_{11}\left(1-k_{2}\right)-a_{1}\left(1-k_{1}\right) \alpha_{21}\right)}{\alpha_{11}^{2} \alpha_{22}}\right) \\
& +\frac{\varepsilon}{d_{22}}\left(a_{2}\left(1-k_{2}\right)\left(d_{21} x_{n}+d_{22} y_{n}+\frac{a_{2} a_{11}\left(1-k_{2}\right)-a_{1}\left(1-k_{1}\right) \alpha_{21}}{\alpha_{11} \alpha_{22}}\right)\right. \\
& -\frac{a_{1}\left(1-k_{1}\right) \alpha_{21}\left(d_{11} x_{n}+\left(a_{1}\left(1-k_{1}\right) / \alpha_{11}\right)\right)\left(d_{21} x_{n}+d_{22} y_{n}+\left(\left(a_{2} a_{11}\left(1-k_{2}\right)-a_{1}\left(1-k_{1}\right) \alpha_{21}\right) / \alpha_{11} \alpha_{22}\right)\right)}{\alpha_{11}} \\
& \left.-\left(d_{21} x_{n}+d_{22} y_{n}+\frac{a_{2} a_{11}\left(1-k_{2}\right)-a_{1}\left(1-k_{1}\right) \alpha_{21}}{\alpha_{11} \alpha_{22}}\right)^{2} \alpha_{22}\right) \\
& +\varepsilon\left(a_{3}\left(x_{n}+y_{n}+z_{n}+\frac{a_{3} \alpha_{11} \alpha_{22}-a_{2}\left(1-k_{2}\right) \alpha_{11} \alpha_{32}+a_{1}\left(1-k_{1}\right) \alpha_{21} \alpha_{32}}{\alpha_{11} \alpha_{22} \alpha_{33}}\right)\right. \\
& -\alpha_{32}\left(d_{21} x_{n}+d_{22} y_{n}+\frac{a_{2} a_{11}\left(1-k_{2}\right)-a_{1}\left(1-k_{1}\right) \alpha_{21}}{\alpha_{11} \alpha_{22}}\right) \\
& \times\left(x_{n}+y_{n}+z_{n}+\frac{a_{3} \alpha_{11} \alpha_{22}-a_{2}\left(1-k_{2}\right) \alpha_{11} \alpha_{32}+a_{1}\left(1-k_{1}\right) \alpha_{21} \alpha_{32}}{\alpha_{11} \alpha_{22} \alpha_{33}}\right) \\
& \left.-\left(x_{n}+y_{n}+z_{n}+\frac{a_{3} \alpha_{11} \alpha_{22}-a_{2}\left(1-k_{2}\right) \alpha_{11} \alpha_{32}+a_{1}\left(1-k_{1}\right) \alpha_{21} \alpha_{32}}{\alpha_{11} \alpha_{22} \alpha_{33}}\right)^{2} \alpha_{33}\right) \text {. }
\end{aligned}
$$


Now, from system (143) on the center manifold

$$
W^{c}(0)=\left\{\left(x_{n}, y_{n}, z_{n}\right) \mid\left(y_{n}, z_{n}\right)=\left(\chi_{7}\left(x_{n}\right), \chi_{8}\left(x_{n}\right)\right), \chi_{i}(0)=0, D \chi_{i}(0)=0, i=7,8\right\},
$$

where

$$
\chi_{i}\left(x_{n}\right)=a_{i} x^{2}+b_{i} x^{3}+O(x)^{4}, \quad \text { for } i=7,8 .
$$

From (143) and (145), we obtain

$$
\begin{aligned}
& \chi_{7}\left(-x_{n}+F_{2}^{*}\left(x_{n}, \chi_{7}, \chi_{8}\right)\right)=\lambda_{2} \chi_{7}\left(x_{n}\right)+G_{2}^{*}\left(x_{n}, \chi_{7}, \chi_{8}\right), \\
& \chi_{8}\left(-x_{n}+F_{2}^{*}\left(x_{n}, \chi_{7}, \chi_{8}\right)\right)=\lambda_{3} \chi_{8}\left(x_{n}\right)+H_{2}^{*}\left(x_{n}, \chi_{7}, \chi_{8}\right) .
\end{aligned}
$$

$$
f_{1}^{*}\left(x_{n}\right)=-x_{n}+\frac{h\left[\left(\left(a_{1}\left(1-k_{1}\right)\right)^{2} / \alpha_{11}\right)-\left(x_{n} d_{11}+\left(a_{1}\left(1-k_{1}\right) / \alpha_{11}\right)\right)^{2} \alpha_{11}\right]+\varepsilon\left[a_{1}\left(1-k_{1}\right)\left(x_{n} d_{11}+\left(a_{1}\left(1-k_{1}\right) / \alpha_{11}\right)\right)-\left(x_{n} d_{11}+\left(a_{1}\left(1-k_{1}\right) / \alpha_{11}\right)\right)^{2} \alpha_{11}\right]}{d_{11}} .
$$

From (117) and (148), the computation yields: $\Omega_{1}=a_{1}\left(1-k_{1}\right)\left(1-2 d_{11}\right) \neq 0$. Moreover, $\Omega_{2}=\left(h \alpha_{11} d_{11}+\right.$ $\left.2 \alpha_{11} d_{11} \varepsilon\right)^{2}>0$. This implies that about $P_{8}$ model (15) undergoes flip bifurcation if $\left.\Omega \in \mathscr{F}\right|_{P_{8}}$. Moreover, period-2 points bifurcating from $P_{8}$ are stable since $\Omega_{2}=$ $\left(h \alpha_{11} d_{11}+2 \alpha_{11} d_{11}\right)^{2}>0$.

\section{Numerical Simulations}

Numerical simulations of three-species model (15) are performed in this section to check previous theoretical findings and to show rich dynamical behaviors. In this regard, following eight cases are presented to address the accuracy of theoretical results obtained about fixed points for model (15):

Case I: if $a_{1}=4.1, a_{2}=4.2, a_{3}=0.08, k_{1}=2.2, k_{2}=0.6$ , $\alpha_{11}=0.04, \alpha_{22}=0.4, \alpha_{21}=0.09, \alpha_{32}=0.09$, and $\alpha_{33}=0.4$, then, from (29), one gets $h=$ 0.4065040650406504. From (27), if $h=0.01$ $<\min \{0.4065040650406504, \quad 0.20703933747412007\}$ and starting from $(0.9,0.1,0.2)$, then Figure 1 (a) indicates that $P_{1}$ of $(15)$ is a source. However, if $h=0.5>\max \{0.406504065040,0.2070393374741\}$,

then Figure 1(b) indicates that $P_{1}$ of (15) is a saddle. Hence, theoretical results obtained in Lemma 2 coincide with numerical simulations.

Case II: if $a_{1}=4.1, a_{2}=4.2, a_{3}=2.7, k_{1}=2.2, k_{2}$ $=0.6, \alpha_{11}=1.2, \alpha_{22}=1.4, \alpha_{21}=1.9, \alpha_{32}=0.9$, and $\alpha_{33}=0.4$, then, from (35), one gets $h=$ 0.7407407407407407 . Figure 2(a) indicates if $h=0.01$ $<0.7407407407407407$, then $P_{2}$ of (15) is a sink. However, if $h=0.95>0.7407407407407407$, then Figure 2(b) indicates that $P_{2}$ is unstable. Moreover, if $h=0.7407407407407407$, then $P_{2}$ exchanges the stability,
From (147), computation yields: $a_{7}=b_{7}=a_{8}=b_{8}=0$. Finally, map (143); restrict to $W^{c}(0)$ as and in fact, flip bifurcation takes place by Theorem 10 . Therefore, the flip bifurcation diagrams are presented in Figure 3. Finally, maximum Lyapunov exponents corresponding to Figure 3 are drawn in Figure 4.

Case III: if $a_{1}=4.1, a_{2}=4.2, a_{3}=2.7, k_{1}=2.2, k_{2}=$ 1.6, $\alpha_{11}=1.2, \alpha_{22}=1.4, \alpha_{21}=1.9, \alpha_{32}=0.9$, and $\alpha_{33}=0.4$, then, from (42), one gets $h=$ 0.7936507936507935 . Hence, $P_{3}$ is stable if $h<$ 0.7936507936507935 , and exchange stability is $h=$ 0.7407407407407407 , and in fact, flip bifurcation takes place by Theorem 11. Therefore, the flip bifurcation diagrams with initial value $(0,0.3,0.1)$ are presented in Figure 5. Finally, maximum Lyapunov exponents corresponding to Figure 5 are drawn in Figure 6.

Case IV: if $a_{1}=5.1, a_{2}=4.2, a_{3}=2.7, k_{1}=0.09, k_{2}=$ $1.6, \alpha_{11}=1.2, \alpha_{22}=1.4, \alpha_{21}=1.9, \alpha_{32}=0.9$, and $\alpha_{33}=$ 0.4 , then, from (48), one gets $h=0.43094160$ 741219567. Hence, $P_{4}$ is stable if $h<0.4309416074$ 1219567, and exchange stability is $h=0.43094160$ 741219567, and in fact, flip bifurcation takes place by Theorem 12. Therefore, the flip bifurcation diagrams with initial value $(0.1,0.2,0.1)$ are presented in Figure 7. Finally, maximum Lyapunov exponents corresponding to Figure 7 are drawn in Figure 8.

Case V: if $a_{1}=7.1, a_{2}=4.2, a_{3}=2.7, k_{1}=1.9, k_{2}=$ $1.6, \alpha_{11}=1.2, \alpha_{22}=1.4, \alpha_{21}=\quad 1.9, \alpha_{32}=0.9$, and $\alpha_{33}=0.4$, then, from (54), one gets $h=0.312989$ 04538341166. Hence, $P_{5}$ is stable if $h<0.312$ 98904538341166, and exchange stability is $h=$ 0.31298904538341166 , and in fact, flip bifurcation takes place by Theorem 13. Therefore, the flip bifurcation diagrams with initial value $(0,0.1,0.1)$ are presented in Figure 9. Finally, maximum Lyapunov exponents corresponding to Figure 9 are drawn in Figure 10. 


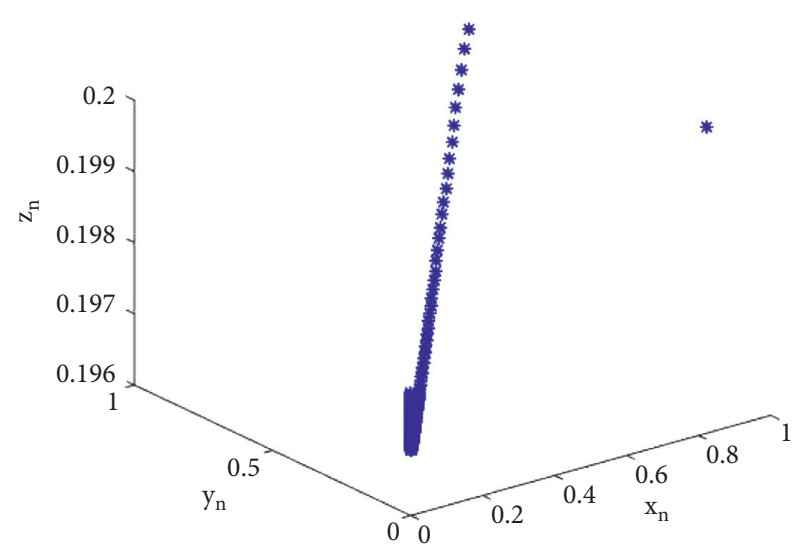

(a)

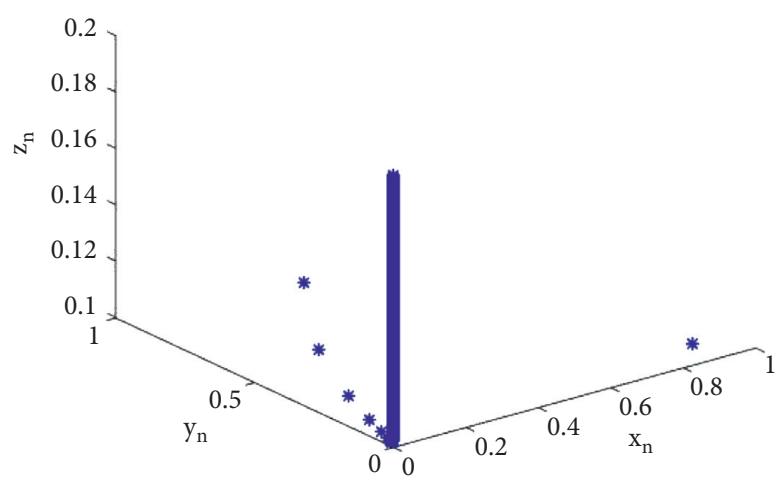

(b)

Figure 1: Phase portrait about $P_{1}$.

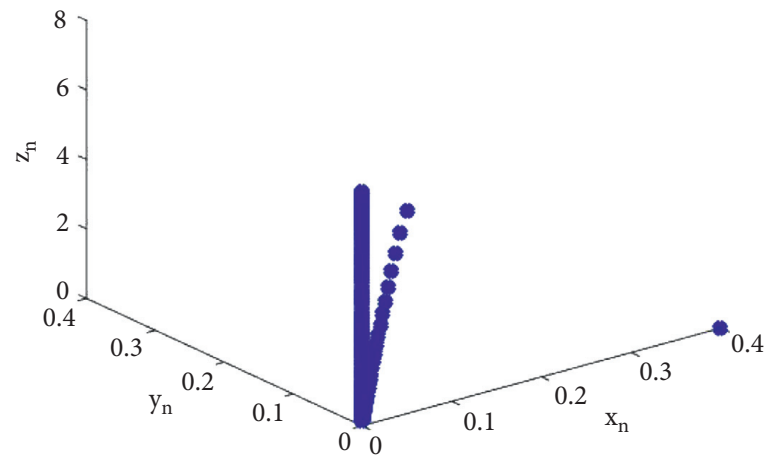

(a)

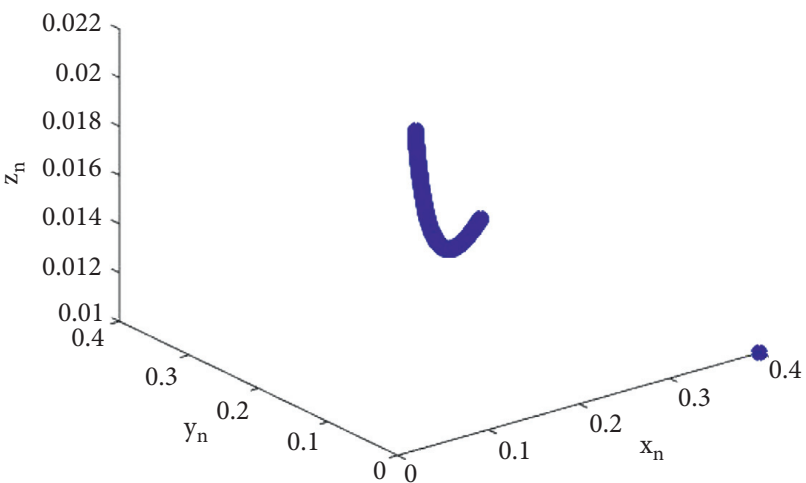

(b)

Figure 2: Phase portrait about $P_{2}$.

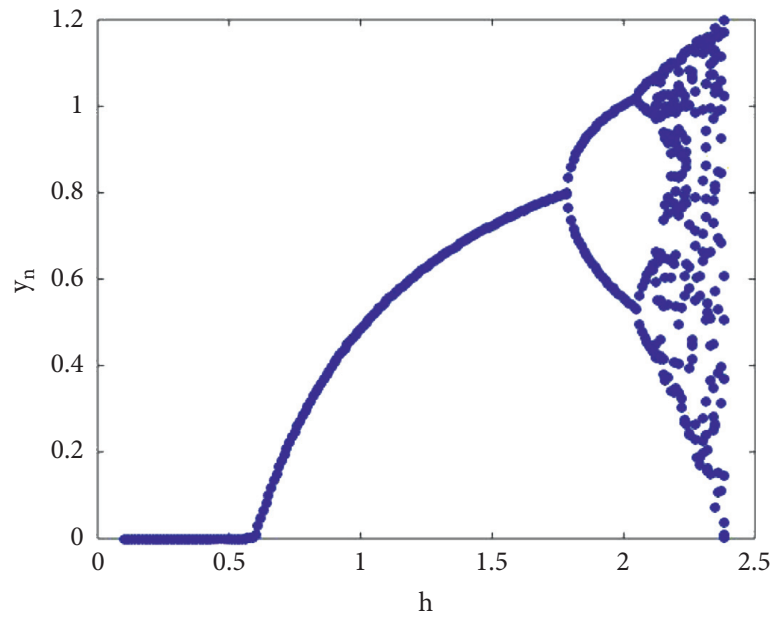

(a)

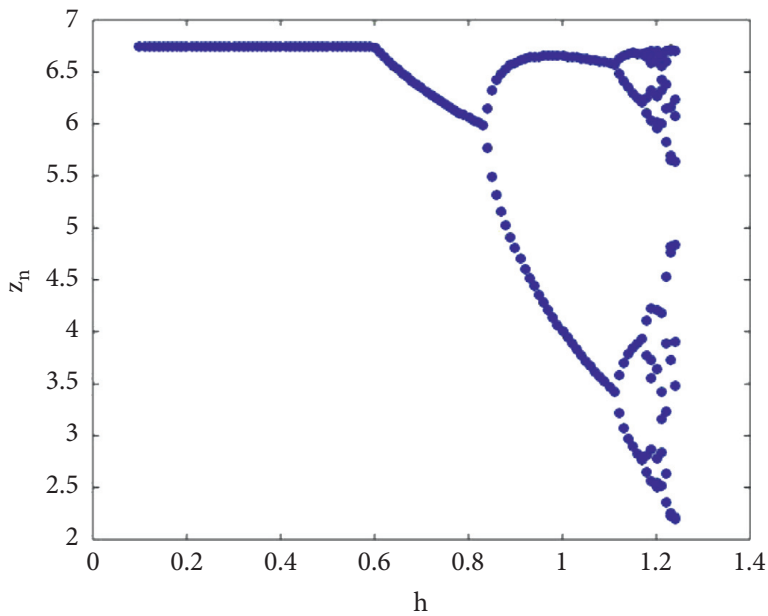

(b)

Figure 3: Continued. 


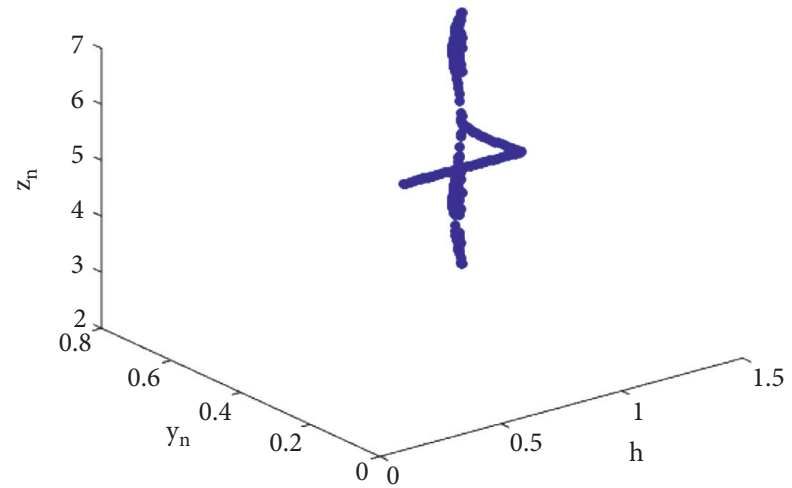

(c)

FIgURE 3: Flip bifurcation diagrams at $P_{2}$, where $h \in[0.1,6.4]$.

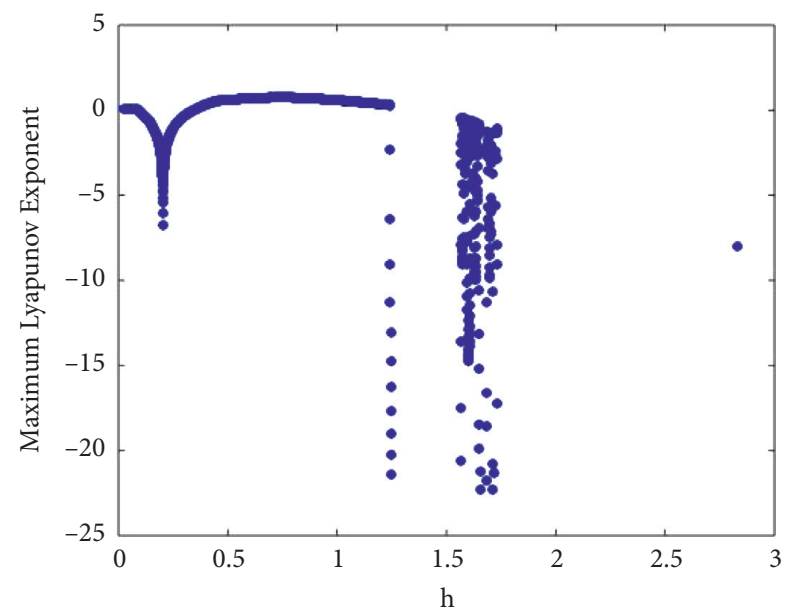

FIgURE 4: Maximum Lyapunov exponents corresponding to Figure 3.

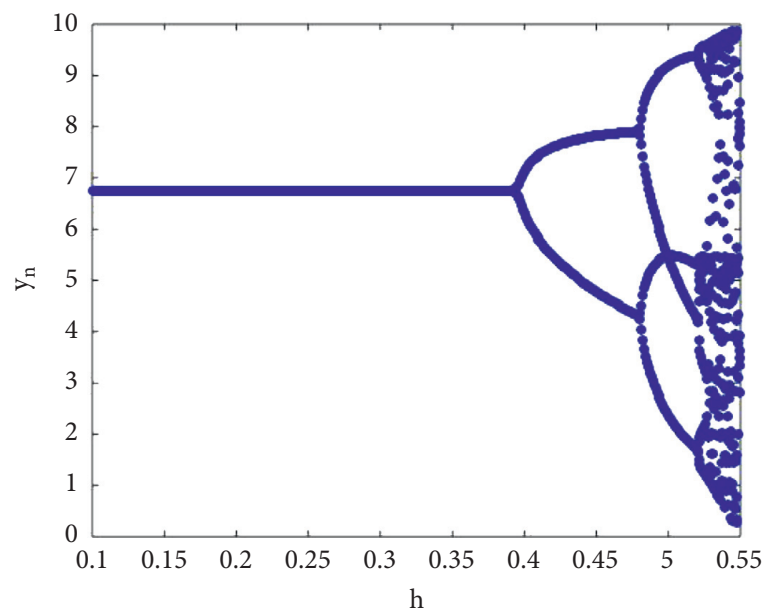

(a)

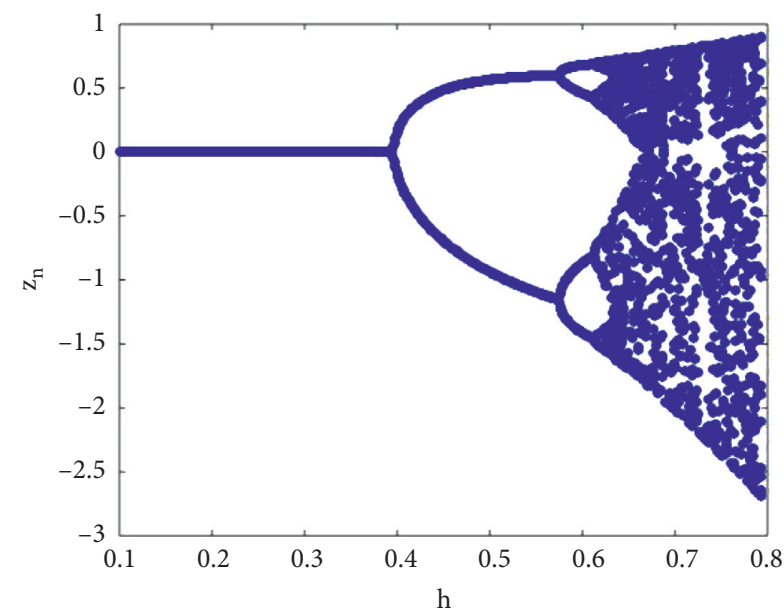

(b)

Figure 5: Continued. 


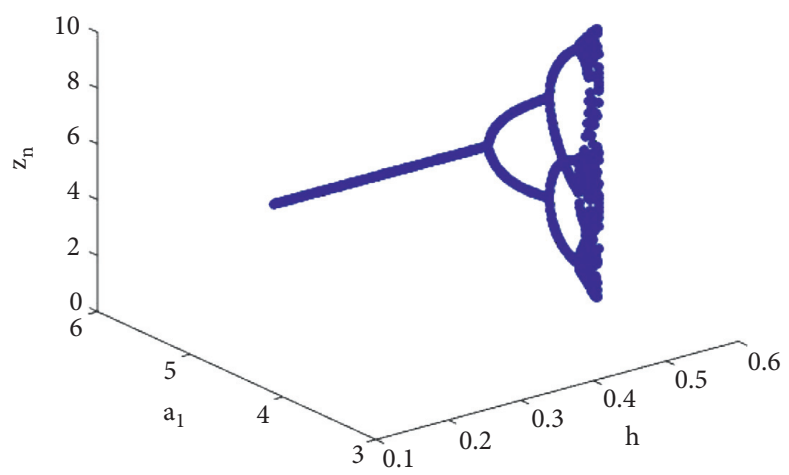

(c)

FIgURE 5: Flip bifurcation diagrams at $P_{3}$, where $h \in[0.1,1.4]$.

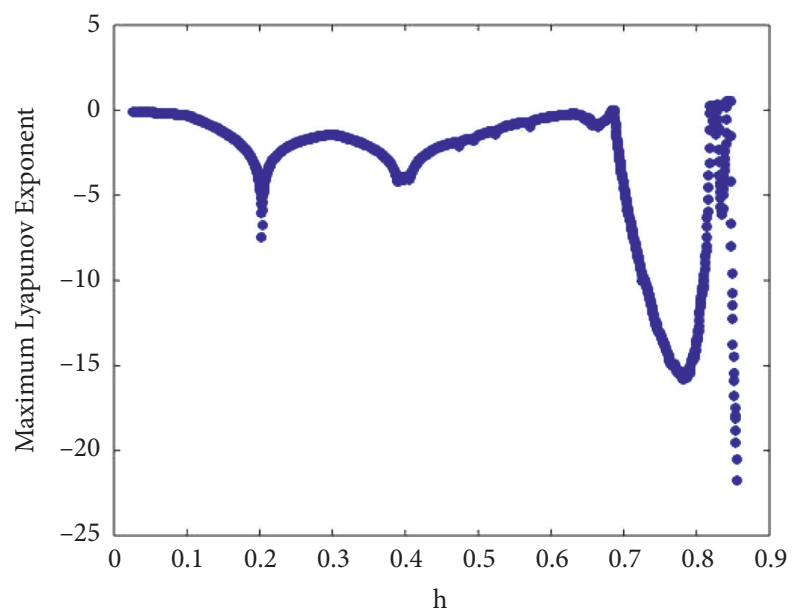

FIgURE 6: Maximum Lyapunov exponents corresponding to Figure 5.

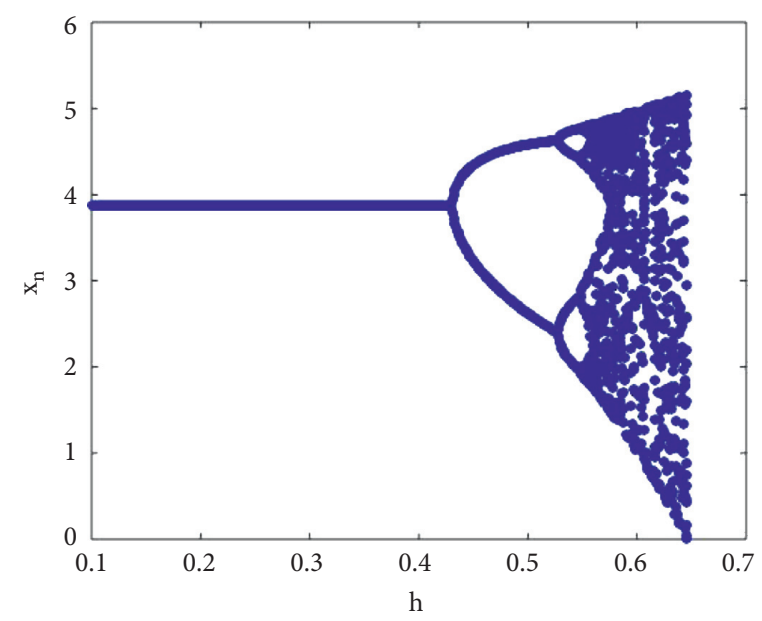

(a)

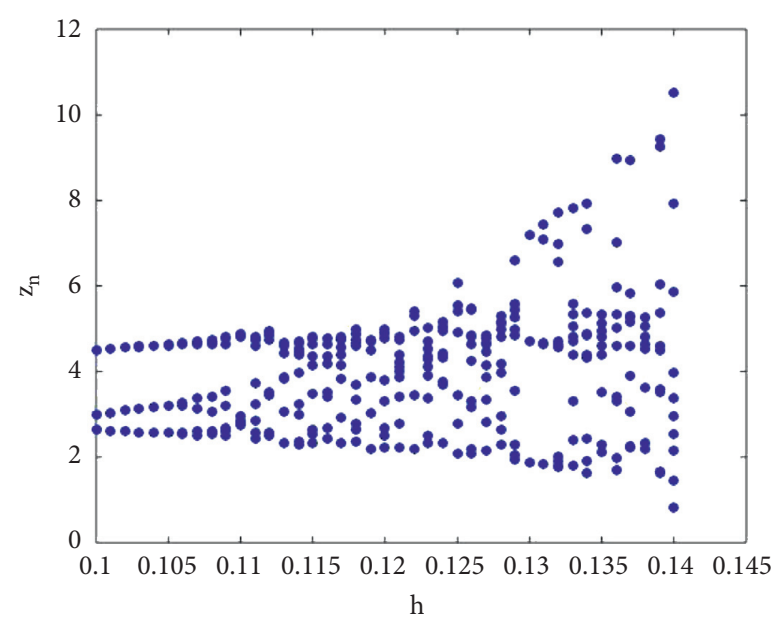

(b)

FIgUre 7: Continued. 


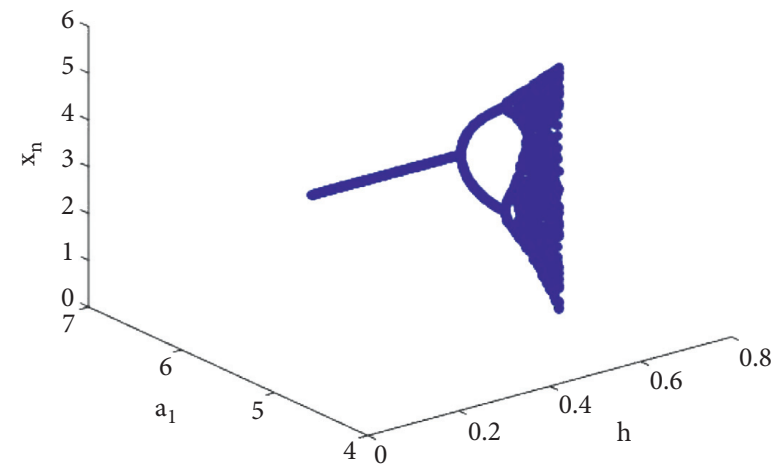

(c)

Figure 7: Flip bifurcation diagrams at $P_{4}$, where $h \in[0: 1 ; 1: 4]$.

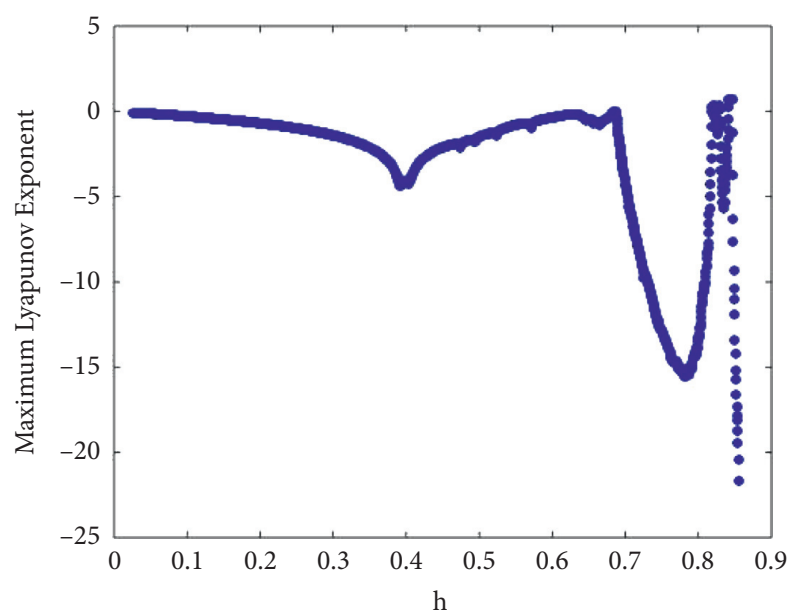

Figure 8: Maximum Lyapunov exponents corresponding to Figure 7.
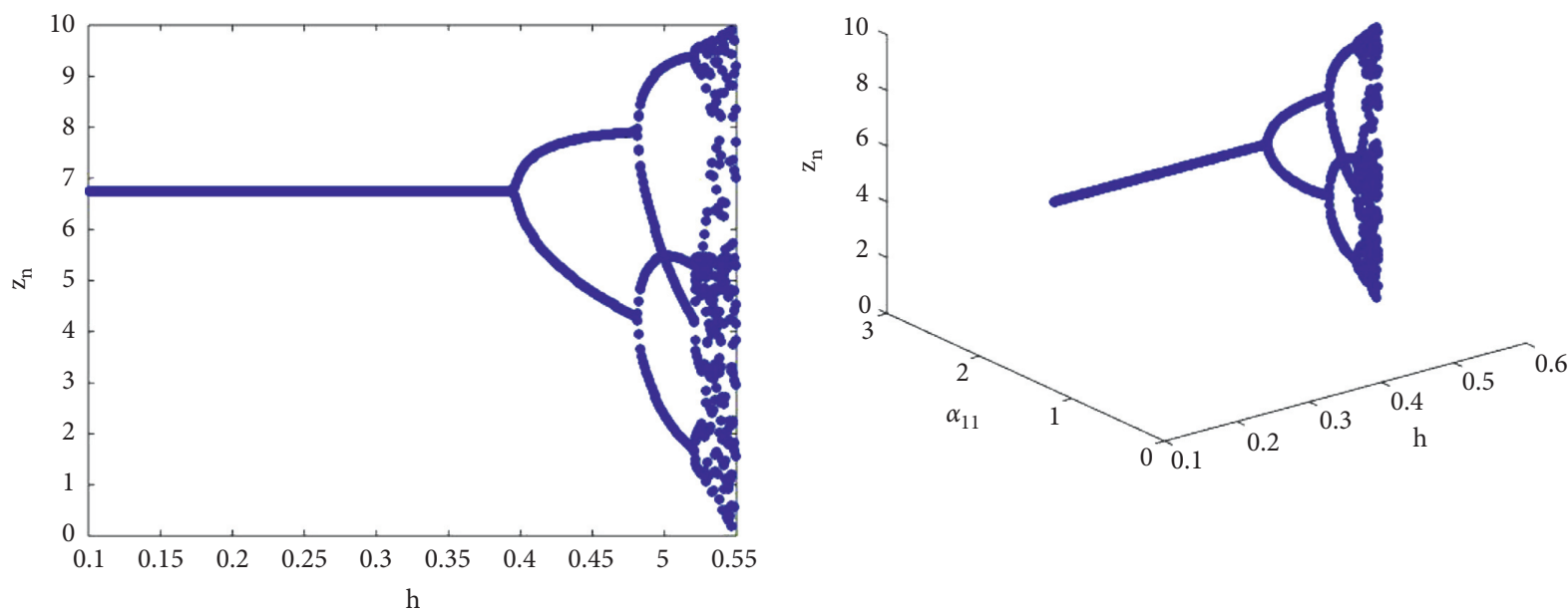

(a)

(b)

FIgURE 9: Continued. 


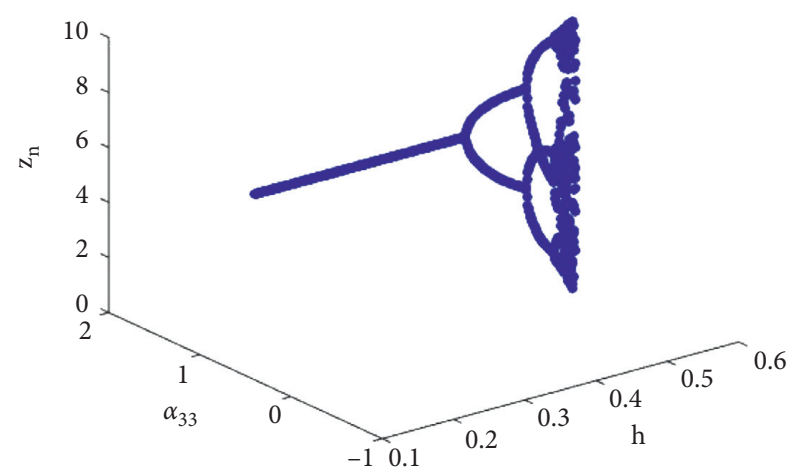

(c)

FIgURE 9: Flip bifurcation diagrams at $P_{5}$, where $h \in[0.1,1.4]$.

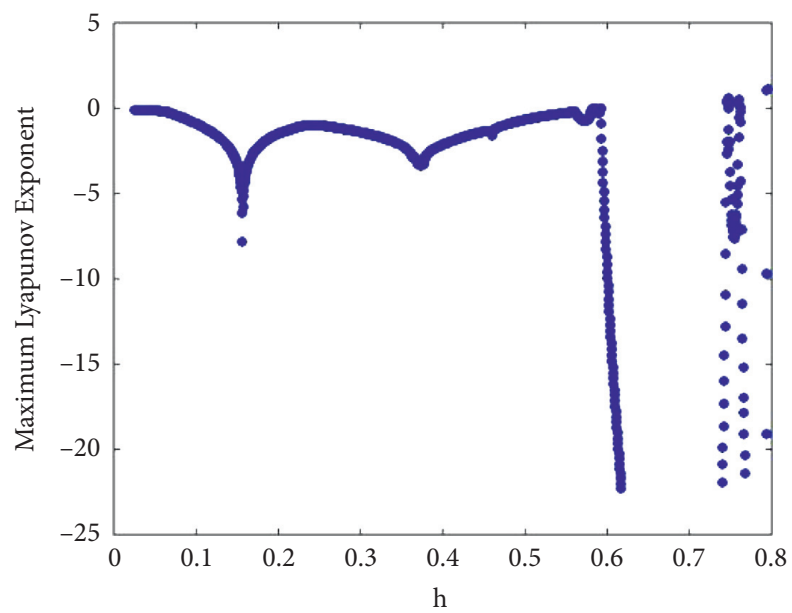

Figure 10: Maximum Lyapunov exponents corresponding to Figure 9.

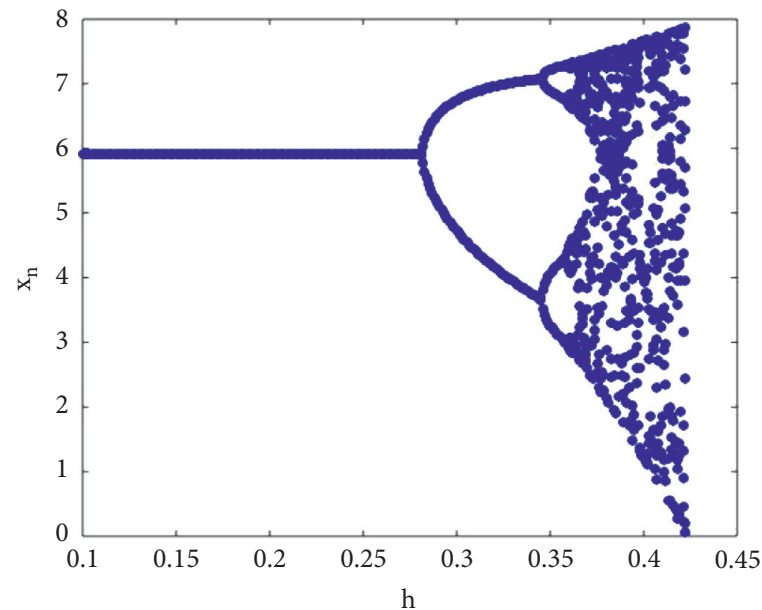

(a)

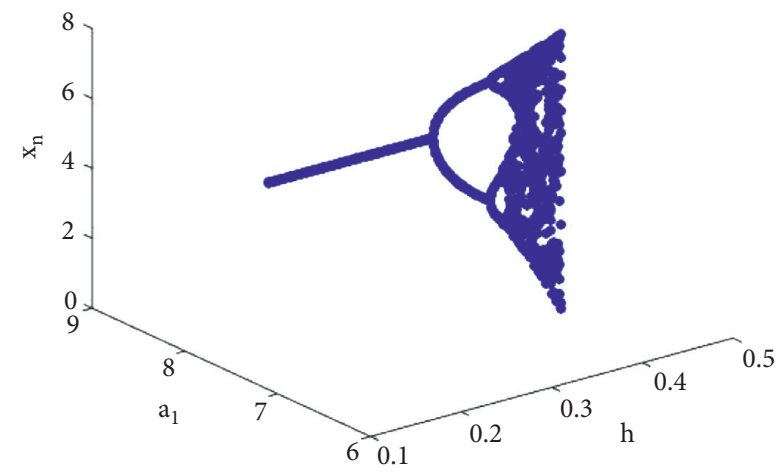

(b)

Figure 11: Continued. 


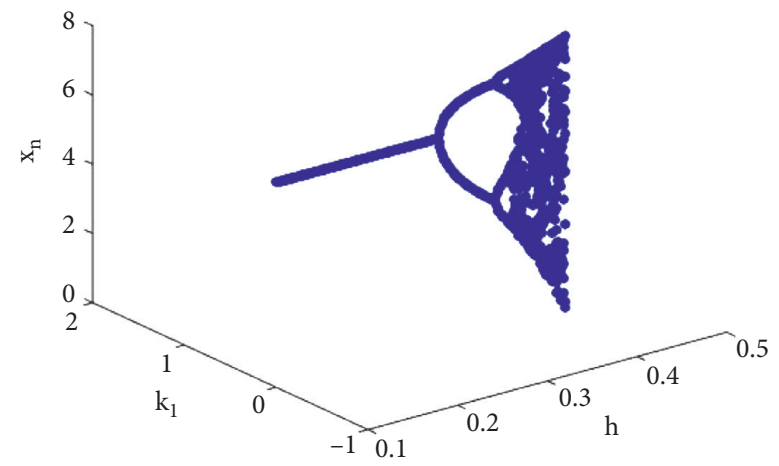

(c)

Figure 11: Flip bifurcation diagrams at $P_{6}$, where $h \in[0.1,1.4]$.

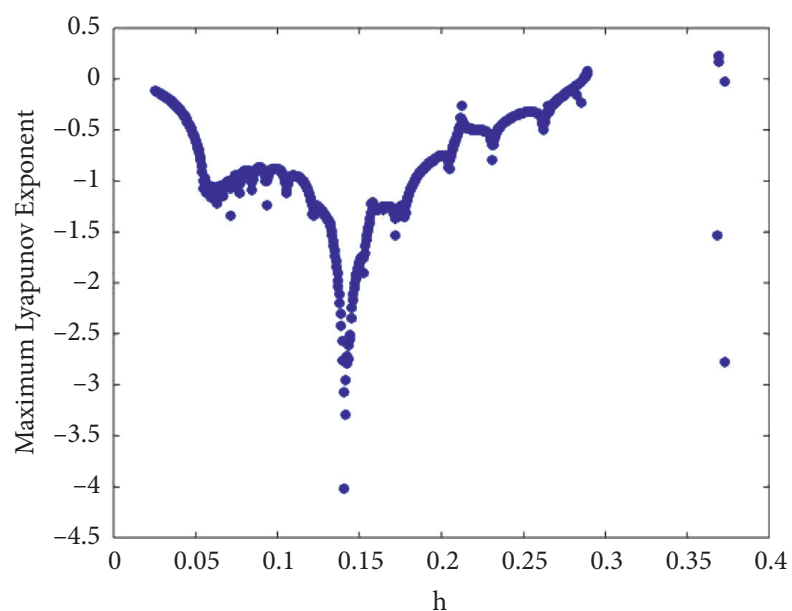

FIgURe 12: Maximum Lyapunov exponents corresponding to Figure 11.

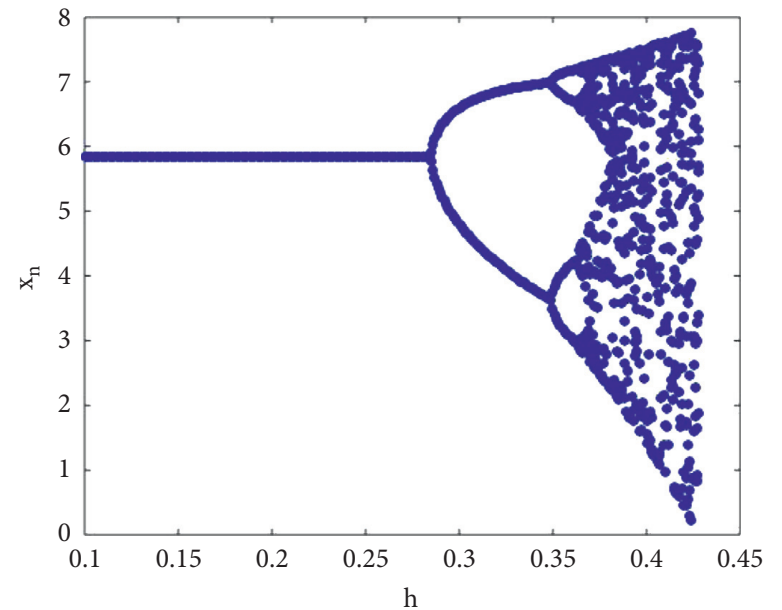

(a)

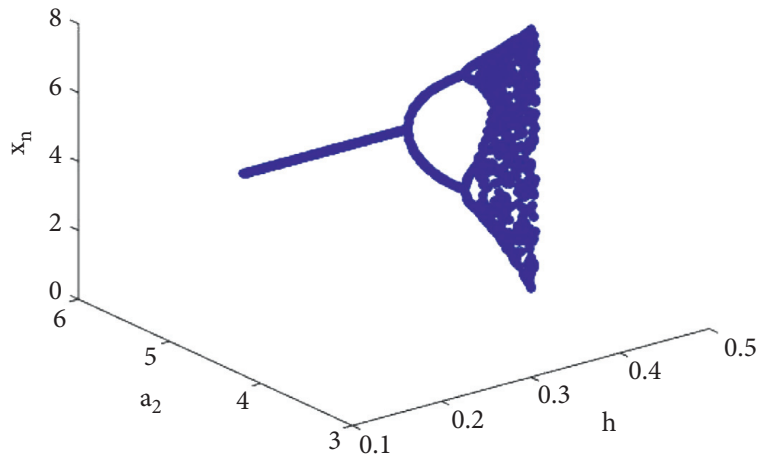

(b)

Figure 13: Continued. 


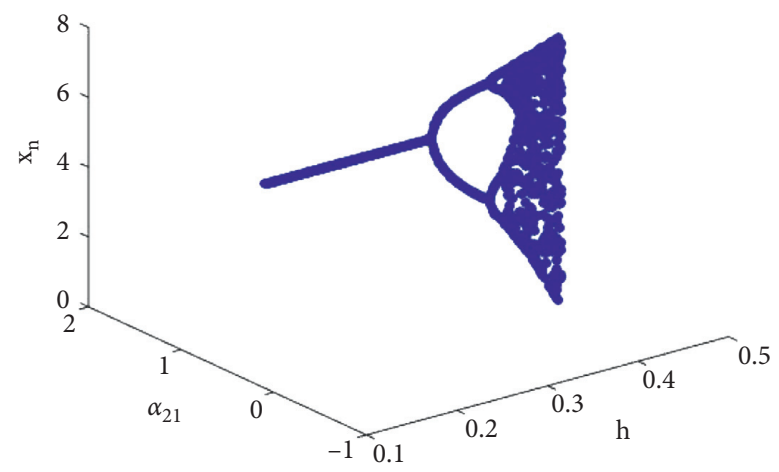

(c)

FIGURE 13: Flip bifurcation diagrams at $P_{7}$, where $h \in[0.1,1.0]$.

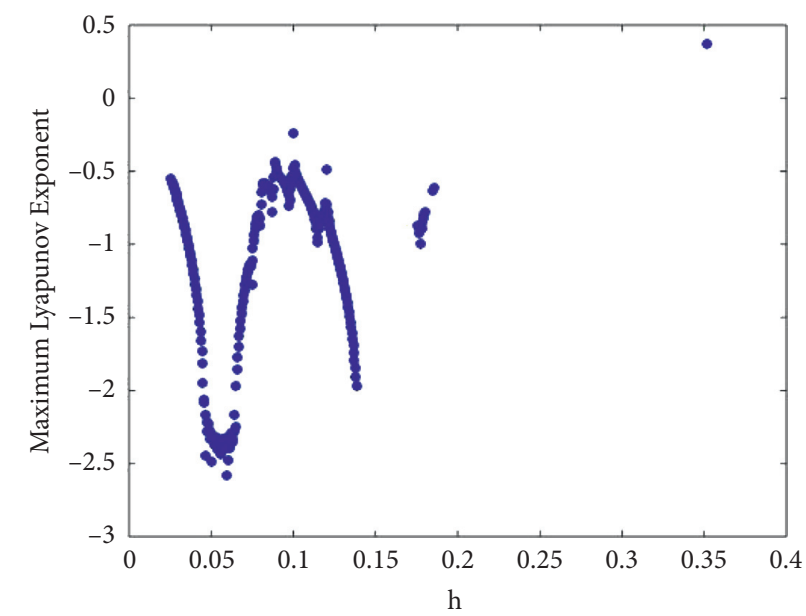

FIgURE 14: Maximum Lyapunov exponents corresponding to Figure 13.

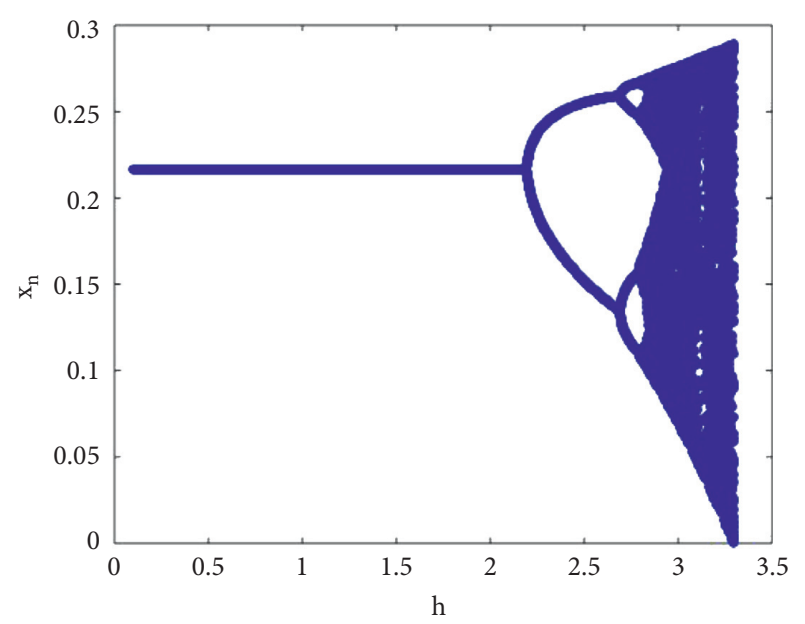

(a)

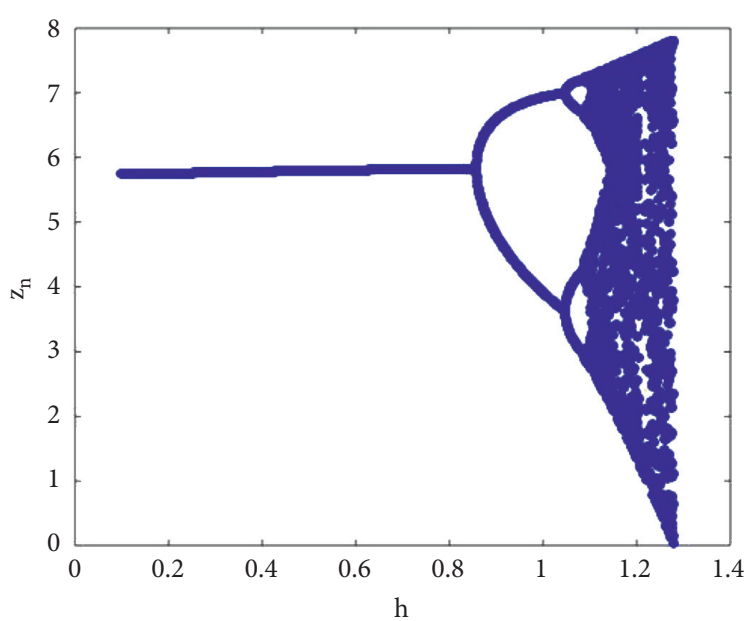

(b)

Figure 15: Continued. 


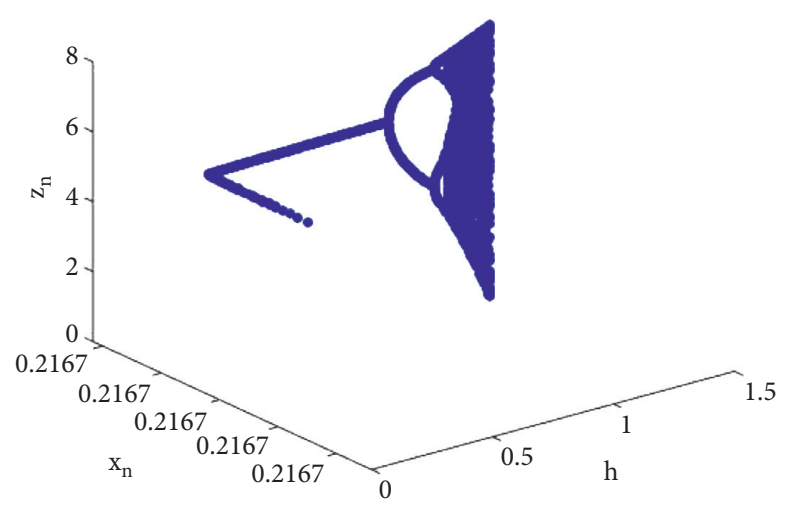

(c)

Figure 15: Flip bifurcation diagrams at $P_{8}$, where $h \in[0.1,4.2]$.

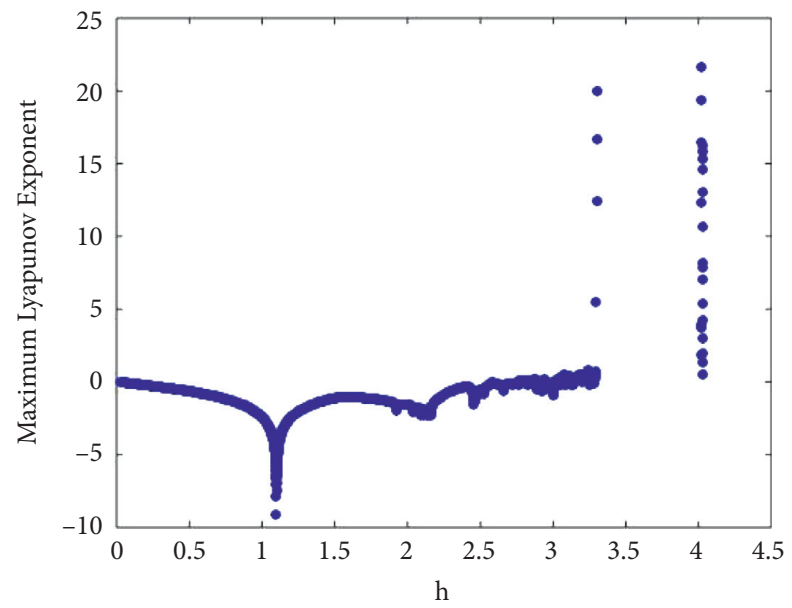

FIgURE 16: Maximum Lyapunov exponents corresponding to Figure 15.

Table 1: Dynamical classifications around fixed points of model (15).

\begin{tabular}{|c|c|}
\hline $\begin{array}{l}\text { Fixed } \\
\text { points }\end{array}$ & Corresponding behavior \\
\hline$P_{1}$ & $\begin{array}{c}\text { Not sink; source if } 0<h<\min \left\{2 / a_{1}\left(k_{1}-1\right), 2 / a_{2}\left(k_{2}-1\right)\right\} ; \text { saddle if } \\
h>\max \left\{2 / a_{1}\left(k_{1}-1\right), 2 / a_{2}\left(k_{2}-1\right)\right\} ; \text { nonhyperbolic if } h=2 / a_{1}\left(k_{1}-1\right) \text { or } h=2 / a_{2}\left(k_{2}-1\right)\end{array}$ \\
\hline$P_{2}$ & $\begin{array}{c}\text { Sink if } h>\max \left\{2 / a_{1}\left(k_{1}-1\right), 2 / a_{2}\left(k_{2}-1\right)\right\} \text { and } 0<h<\left(2 / a_{3}\right) \\
\text { Source if } 0<h<\min \left\{2 / a_{1}\left(k_{1}-1\right), 2 / a_{2}\left(k_{2}-1\right)\right\} \text { and } h>\left(2 / a_{3}\right) \\
\text { Saddle if } 0<h<\min \left\{2 / a_{1}\left(k_{1}-1\right), 2 / a_{2}\left(k_{2}-1\right)\right\} \text { and } 0<h<\left(2 / a_{3}\right) \\
\text { Nonhyperbolic if } h=\left(2 / a_{3}\right) \text { or } h=\left(2 / a_{1}\left(k_{1}-1\right)\right) \text { or } h=\left(2 / a_{2}\left(k_{2}-1\right)\right)\end{array}$ \\
\hline$P_{3}$ & $\begin{array}{l}\text { Sink if } h>\max \left\{2 / a_{1}\left(k_{1}-1\right), 2 \alpha_{22} /\left(a_{2} \alpha_{32}\left(1-k_{2}\right)-a_{3} \alpha_{22}\right)\right\} \text { and } 0<h<2 / a_{2}\left(1-k_{2}\right) \\
\text { Source if } 0<h<\min \left\{2 / a_{1}\left(k_{1}-1\right), 2 \alpha_{22} /\left(a_{2} \alpha_{32}\left(1-k_{2}\right)-a_{3} \alpha_{22}\right)\right\} \text { and } h>2 / a_{2}\left(1-k_{2}\right) \\
\text { Saddle if } h>\max \left\{2 / a_{1}\left(k_{1}-1\right), 2 / a_{2}\left(1-k_{2}\right)\right\} \text { and } 0<h<\left(2 \alpha_{22} /\left(a_{2} \alpha_{32}\left(1-k_{2}\right)-a_{3} \alpha_{22}\right)\right) \\
\text { Nonhyperbolic if } h=2 / a_{2}\left(1-k_{2}\right) \text { or } h=2 / a_{1}\left(k_{1}-1\right) \text { or } h=2 \alpha_{22} /\left(a_{2} \alpha_{32}\left(1-k_{2}\right)-a_{3} \alpha_{22}\right)\end{array}$ \\
\hline$P_{4}$ & $\begin{array}{c}\text { Not sink; source if } h>\max \left\{2 / a_{1}\left(1-k_{1}\right), 2 / a_{2}\left(k_{2}-1\right)\right\} \\
\text { Saddle if } 0<h<\min \left\{2 / a_{1}\left(1-k_{1}\right), 2 / a_{2}\left(k_{2}-1\right)\right\} \\
\text { Nonhyperbolic if } h=\left(2 / a_{1}\left(1-k_{1}\right)\right) \text { or } h=\left(2 / a_{2}\left(k_{2}-1\right)\right)\end{array}$ \\
\hline$P_{5}$ & $\begin{array}{l}\text { Sink if } h>\max \left\{2 / a_{1}\left(k_{1}-1\right), 2 \alpha_{22} /\left(a_{3} \alpha_{22}-a_{2} \alpha_{32}\left(1-k_{2}\right)\right)\right\} \text { and } 0<h<2 / a_{2}\left(1-k_{2}\right) \\
\text { Source if } 0<h<\min \left\{2 / a_{1}\left(k_{1}-1\right), 2 \alpha_{22} /\left(a_{3} \alpha_{22}-a_{2} \alpha_{32}\left(1-k_{2}\right)\right)\right\} \text { and } h>2 / a_{2}\left(1-k_{2}\right) \\
\text { Saddle if } h>\max \left\{2 / a_{1}\left(k_{1}-1\right), 2 \alpha_{22} /\left(a_{3} \alpha_{22}-a_{2} \alpha_{32}\left(1-k_{2}\right)\right)\right\} \text { and } h>2 / a_{2}\left(1-k_{2}\right) \\
\text { Nonhyperbolic if } h=2 / a_{1}\left(k_{1}-1\right) \text { or } h=\left(a_{3} \alpha_{22}-2 \alpha_{22}\right) / a_{2} \alpha_{32}\left(1-k_{2}\right) \text { or } h=2 / a_{2}\left(1-k_{2}\right)\end{array}$ \\
\hline
\end{tabular}


TABle 1: Continued.

\begin{tabular}{|c|c|}
\hline $\begin{array}{l}\text { Fixed } \\
\text { points }\end{array}$ & Corresponding behavior \\
\hline$P_{6}$ & $\begin{array}{c}\text { Sink if } 0<h<\min \left\{2 / a_{1}\left(1-k_{1}\right), 2 / a_{3}\right\} \text { and } h>2 \alpha_{11} /\left(a_{1} \alpha_{21}\left(1-k_{1}\right)-a_{2} \alpha_{11}\left(1-k_{2}\right)\right) \\
\text { source if } h>\max \left\{2 / a_{1}\left(1-k_{1}\right), 2 / a_{3}\right\} \text { and } 0<h<2 \alpha_{11} /\left(a_{1} \alpha_{21}\left(1-k_{1}\right)-a_{2} \alpha_{11}\left(1-k_{2}\right)\right) \\
\text { saddle if } 0<h<\min \left\{2 / a_{1}\left(1-k_{1}\right), 2 / a_{3}\right\} \text { and } 0<h<2 \alpha_{11} /\left(a_{1} \alpha_{21}\left(1-k_{1}\right)-a_{2} \alpha_{11}\left(1-k_{2}\right)\right) \\
\text { nonhyperbolic if } h=2 / a_{1}\left(1-k_{1}\right) \text { or } h=\left(2 / a_{3}\right) \text { or } h=2 \alpha_{11} /\left(a_{1} \alpha_{21}\left(1-k_{1}\right)-a_{2} \alpha_{11}\left(1-k_{2}\right)\right)\end{array}$ \\
\hline$P_{7}$ & $\begin{array}{c}\text { Sink if } 0<h<\min \left\{2 / a_{1}\left(1-k_{1}\right), 2 \alpha_{11} /\left(a_{2} \alpha_{11}\left(1-k_{2}\right)-2 a_{1} \alpha_{21}\left(1-k_{1}\right)\right)\right\} \text { and } \\
h>2 \alpha_{11} \alpha_{22} /\left(a_{3} \alpha_{11} \alpha_{22}-a_{2} \alpha_{11} \alpha_{32}\left(1-k_{2}\right)+a_{1} \alpha_{21} \alpha_{32}\left(1-k_{1}\right)\right) \\
\text { Source if } h>\max \left\{2 / a_{1}\left(1-k_{1}\right), 2 \alpha_{11} /\left(a_{2} \alpha_{11}\left(1-k_{2}\right)-2 a_{1} \alpha_{21}\left(1-k_{1}\right)\right)\right\} \text { and } \\
0<h<2 \alpha_{11} \alpha_{22} /\left(a_{3} \alpha_{11} \alpha_{22}-a_{2} \alpha_{11} \alpha_{32}\left(1-k_{2}\right)+a_{1} \alpha_{21} \alpha_{32}\left(1-k_{1}\right)\right) \\
\text { Saddle if } 0<h<\min \left\{2 / a_{1}\left(1-k_{1}\right), 2 \alpha_{11} /\left(a_{2} \alpha_{11}\left(1-k_{2}\right)-2 a_{1} \alpha_{21}\left(1-k_{1}\right)\right)\right\} \text { and } \\
h<2 \alpha_{11} \alpha_{22} /\left(a_{3} \alpha_{11} \alpha_{22}-a_{2} \alpha_{11} \alpha_{32}\left(1-k_{2}\right)+a_{1} \alpha_{21} \alpha_{32}\left(1-k_{1}\right)\right) \\
\text { Nonhyperbolic if } h=2 / a_{1}\left(1-k_{1}\right) \text { or } h=2 \alpha_{11} /\left(a_{2} \alpha_{11}\left(1-k_{2}\right)-2 a_{1} \alpha_{21}\left(1-k_{1}\right)\right) \text { or } \\
h=2 \alpha_{11} \alpha_{22} /\left(a_{3} \alpha_{11} \alpha_{22}-a_{2} \alpha_{11} \alpha_{32}\left(1-k_{2}\right)+a_{1} \alpha_{21} \alpha_{32}\left(1-k_{1}\right)\right)\end{array}$ \\
\hline$P_{8}$ & $\begin{array}{l}\text { Sink if } \\
\begin{array}{l}0<h<\min \left\{2 / a_{1}\left(1-k_{1}\right), 2 \alpha_{11} /\left(a_{2} \alpha_{11}\left(1-k_{2}\right)-2 a_{1} \alpha_{21}\left(1-k_{1}\right)\right), 2 \alpha_{11} \alpha_{22} /\left(a_{3} \alpha_{11} \alpha_{22}-a_{2} \alpha_{11} \alpha_{32}\left(1-k_{2}\right)+a_{1} \alpha_{21} \alpha_{32}\left(1-k_{1}\right)\right)\right\} \\
\text { Source if }\end{array} \\
h>\max \left\{2 / a_{1}\left(1-k_{1}\right), 2 \alpha_{11} /\left(a_{2} \alpha_{11}\left(1-k_{2}\right)-2 a_{1} \alpha_{21}\left(1-k_{1}\right)\right), 2 \alpha_{11} \alpha_{22} /\left(a_{3} \alpha_{11} \alpha_{22}-a_{2} \alpha_{11} \alpha_{32}\left(1-k_{2}\right)+a_{1} \alpha_{21} \alpha_{32}\left(1-k_{1}\right)\right)\right\} \\
\text { Saddle if } 0<h<\min \left\{2 / a_{1}\left(1-k_{1}\right), 2 \alpha_{11} /\left(a_{2} \alpha_{11}\left(1-k_{2}\right)-2 a_{1} \alpha_{21}\left(1-k_{1}\right)\right)\right\} \text { and } \\
\qquad h>2 \alpha_{11} \alpha_{22} /\left(a_{3} \alpha_{11} \alpha_{22}-a_{2} \alpha_{11} \alpha_{32}\left(1-k_{2}\right)+a_{1} \alpha_{21} \alpha_{32}\left(1-k_{1}\right)\right)\end{array}$ \\
\hline
\end{tabular}

Case VI: if $a_{1}=7.1, a_{2}=4.2, a_{3}=2.7, k_{1}=0.0009, k_{2}=$ $1.6, \alpha_{11}=1.2, \alpha_{22}=1.4, \alpha_{21}=1.9, \alpha_{32}=0.9, \alpha_{33}=0.4$ then from (61) one gets: $h=0.2819438903463822$. Hence $P_{6}$ is stable if $h<0.2819438903463822$, and exchange stability if $h=0.2819438903463822$ and infact flip bifurcation takes place by Theorem 14 . Therefore the flip bifurcation diagrams with initial value $(0.1,0,0.1)$ are presented in Figure 11 . Finally maximum lypunov exponents corresponding to Figure 11 are drawn in Figure 12.

Case VII: If $a_{1}=9.1, a_{2}=4.2, a_{3}=2.7, k_{1}=0.23, k_{2}=$ 4.6, $\alpha_{11}=1.2, \alpha_{22}=1.4, \alpha_{21}=0.099, \alpha_{32}=5.9$, and $\alpha_{33}$ $=2.4$, then, from (68), one gets $h=0.285428$ 8568574283. Hence, $P_{7}$ is stable if $h<0.2854$ 288568574283, and exchange stability is $h=0.2854$ 288568574283, and in fact, flip bifurcation takes place by Theorem 15 . Therefore, the flip bifurcation diagrams with initial value $(0.2,0.1,0)$ are presented in Figure 13. Finally, maximum Lyapunov exponents corresponding to Figure 13 are drawn in Figure 14.

Case VIII: if $a_{1}=9.1, a_{2}=1.2, a_{3}=2.7, k_{1}=0.9, k_{2}=$ $0.006, \alpha_{11}=4.2, \alpha_{22}=1.4, \alpha_{21}=4.9, \alpha_{32}=1.9$, and $\alpha_{33}=0.4$, then, from (75), one gets $h=2.19780$ 21978021984. Hence, $P_{7}=(0.21666666666666$, $0.09366666666666,6.305083333333)$ is stable if $h<$ 2.1978021978021984, and exchange stability is $h=2.1978021978021984$, and in fact, flip bifurcation takes place by Theorem 16. Therefore, flip bifurcation diagrams with $(0.2,0.2,0.4)$ are presented in Figure 15 which indicates that period-2 points bifurcate from $P_{8}$ are stable, since $\Omega_{2}=\left(h \alpha_{11} d_{11}+2 \alpha_{11} d_{11}\right)^{2}=$ $310.844023668>0$. Finally, maximum Lyapunov exponents corresponding to Figure 15 are drawn in Figure 16.

\section{Conclusion}

The work is about the existence of fixed points, topological classifications around fixed points, periodic points, and bifurcations of a three-species discrete food chain model with harvesting in the region: $\mathbb{R}_{+}^{3}=\{(x, y, z): x, y, z \geq 0\}$. We proved that, for all parametric values $h, a_{1}, a_{2}, a_{3}, k_{1}, k_{2}$, $\alpha_{11}, \alpha_{22}, \alpha_{21}, \alpha_{32}$, and $\alpha_{33}$, model (15) has trivial fixed point: $P_{1}=(0,0,0)$; boundary fixed points: $P_{2}=\left(0,0, a_{3} / \alpha_{33}\right)$ $\forall a_{3}, \alpha_{33}>0 ; P_{3}=\left(0,\left(1-k_{2}\right) a_{2} / \alpha_{22}, 0\right)$ if $k_{2}<1 ; P_{4}=((1-$ $\left.\left.k_{1}\right) a_{1} / \alpha_{11}, 0,0\right)$ if $k_{1}<1 ; \quad P_{5}=\left(0,\left(1-k_{2}\right) a_{2} / \alpha_{22},\left(\left(a_{3} \alpha_{22}-\right.\right.\right.$ $\left.\left.\left.\alpha_{32}\left(1-k_{2}\right) a_{2}\right) / \alpha_{22} \alpha_{33}\right)\right)$ if $a_{3}>\left(\alpha_{32}\left(1-k_{2}\right) a_{2} / \alpha_{22}\right)$ with $k_{2}$ $<1 ; P_{6}=\left(\left(1-k_{1}\right) a_{1} / \alpha_{11}, 0, a_{3} / \alpha_{33}\right)$ if $k_{1}<1 ; P_{7}=\left(\left(1-k_{1}\right)\right.$ $\left.a_{1} / \alpha_{11},\left(a_{2}\left(1-k_{2}\right) \alpha_{11}-a_{1}\left(1-k_{1}\right) \alpha_{21}\right) / \alpha_{11} \alpha_{22}, 0\right)$ if $a_{2}>\left(a_{1}\right.$ $\left.\left(1-k_{1}\right) \alpha_{21} /\left(1-k_{2}\right) \alpha_{11}\right)$ with $k_{1}, k_{2}<1$. We also proved that if $k_{1}<1, a_{2}>\left(a_{1}\left(1-k_{1}\right) \alpha_{21} /\left(1-k_{2}\right) \alpha_{11}\right)$ and $a_{3}>\left(a_{2}(1\right.$ $\left.\left.-k_{2}\right) \alpha_{11} \alpha_{32}-a_{1}\left(1-k_{1}\right) \alpha_{21} \alpha_{32}\right) / \alpha_{11} \alpha_{22}$; then, $P_{8}=\left(\left(\left(1-k_{1}\right)\right.\right.$ $\left.a_{1} / \alpha_{11}\right),\left(a_{2}\left(1-k_{2}\right) \alpha_{11}-a_{1}\left(1-k_{1}\right) \alpha_{21}\right) / \alpha_{11} \alpha_{22},\left(a_{3} \alpha_{11} \alpha_{22}-\right.$ $\left.\left.a_{2}\left(1-k_{2}\right) \alpha_{11} \alpha_{32}+a_{1}\left(1-k_{1}\right) \alpha_{21} \alpha_{32}\right) / \alpha_{11} \alpha_{22} \alpha_{33}\right)$ is an interior equilibrium point of (15). Furthermore, we studied the local stability with different topological classifications around each fixed points whose main findings are presented in Table 1. Next, for under consideration model (15), we also studied existence of periodic points by existing theory. Furthermore, we explored the existence of possible bifurcations about each fixed points in order to understand dynamics of model (15) deeply. It is proved that (i) around $P_{1}$ model undergoes no flip bifurcation if $\left.\Omega \in \mathscr{F}\right|_{P_{1}}=$ $\left\{\Omega: h=2 / a_{1}\left(k_{1}-1\right)\right\}$, (ii) around $P_{2}$ model undergoes flip bifurcation if $\left.\Omega \in \mathscr{F}\right|_{P_{2}}=\left\{\Omega: h=2 / a_{3}\right\}$, (iii) around $P_{3}$ model undergoes flip bifurcation if $\left.\Omega \in \mathscr{F}\right|_{P_{3}}=\{\Omega: h=$ $\left.2 / a_{2}\left(1-k_{2}\right)\right\}$, (iv) around $P_{4}$ model undergoes flip bifurcation if $\left.\Omega \in \mathscr{F}\right|_{P_{4}}=\left\{\Omega: h=2 / a_{1}\left(1-k_{1}\right)\right\}$, (v) around $P_{5}$ model undergoes flip bifurcation if $\left.\Omega \in \mathscr{F}\right|_{P_{5}}=\{\Omega: h=$ $\left.2 / a_{1}\left(k_{1}-1\right)\right\}, \quad(v i)$ around $P_{6}$ model undergoes flip 
bifurcation if $\left.\Omega \in \mathscr{F}\right|_{P_{6}}=\left\{\Omega: h=2 / a_{1}\left(1-k_{1}\right)\right\}$, (vii) around $P_{7}$ model undergoes flip bifurcation if $\left.\Omega \in \mathscr{F}\right|_{P_{7}}=$ $\left\{\Omega: h=2 / a_{1}\left(1-k_{1}\right)\right\}$, and (viii) around $P_{8}$ model undergoes flip bifurcation if $\left.\Omega \in \mathscr{F}\right|_{P_{8}}=\left\{\Omega: h=2 / a_{1}\left(1-k_{1}\right)\right\}$. Finally, obtained results are verified numerically. This research can provide a framework for theoretical basis and help for the research in different aspects of biology specifically in the field of ecology.

\section{Data Availability}

All the data used in this study are included within the article and the sources from where they were adopted are cited accordingly.

\section{Conflicts of Interest}

The authors declare that they have no conflicts of interest.

\section{References}

[1] A. J. Lotka, Elements of Physical Biology, Williams \& Wilkins, Philadelphia, PA, USA, 1925.

[2] V. Volterra, Leconssen la theory of mathematique de leitte pou lavi, Gautheir-Villars, Paris, France, 1931.

[3] J. R. Beddington, C. A. Free, and J. H. Lawton, "Dynamic complexity in predator-prey models framed in difference equations," Nature, vol. 255, no. 5503, pp. 58-60, 1975.

[4] F. Chen, "Permanence and global attractivity of a discrete multispecies Lotka-Volterra competition predator-prey systems," Applied Mathematics and Computation, vol. 182, no. 1, pp. 3-12, 2006.

[5] N. Fang and X. X. Chen, "Permanence of a discrete multispecies Lotka-Volterra competition predator-prey system with delays," Nonlinear Analysis: Real World Applications, vol. 9, no. 5, pp. 2185-2195, 2008.

[6] Q. Fang, X. Li, and M. Cao, "Dynamics of a discrete predatorprey system with Beddington-DeAngelis function response," Applied Mathematics, vol. 3, no. 4, pp. 389-394, 2012.

[7] H. N. Agiza, E. M. Elabbasy, H. El-Metwally, and A. A. Elsadany, "Chaotic dynamics of a discrete prey-predator model with Holling type II," Nonlinear Analysis: Real World Applications, vol. 10, no. 1, pp. 116-129, 2009.

[8] H. F. Huo and W. T. Li, "Stable periodic solution of the discrete periodic Leslie-Gower predator-prey model," Mathematical and Computer Modelling, vol. 40, no. 3-4, pp. 261269, 2004.

[9] C. Lu and L. Zhang, "Permanence and global attractivity of a discrete semi-ratio dependent predator-prey system with Holling II type functional response," Journal of Applied Mathematics and Computing, vol. 33, no. 1, pp. 125-135, 2010.

[10] M. Zhao and L. Zhang, "Permanence and chaos in a hostparasitoid model with prolonged diapause for the host," Communications in Nonlinear Science and Numerical Simulation, vol. 14, no. 12, pp. 4197-4203, 2009.

[11] M. Zhao, L. Zhang, and J. Zhu, "Dynamics of a host-parasitoid model with prolonged diapause for parasitoid," Communications in Nonlinear Science and Numerical Simulation, vol. 16, no. 1, pp. 455-462, 2011.

[12] S. M. Salman, A. M. Yousef, and A. A. Elsadany, "Stability, bifurcation analysis and chaos control of a discrete predatorprey system with square root functional response," Chaos, Solitons \& Fractals, vol. 93, pp. 20-31, 2016.
[13] X. Liu and D. Xiao, "Complex dynamic behaviors of a discrete-time predator-prey system," Chaos, Solitons \& Fractals, vol. 32, no. 1, pp. 80-94, 2007.

[14] K. A. Hasan and M. F. Hama, "Complex dynamics behaviors of a discrete prey-predator model with Beddington-Deangelis functional response," International Journal of Contemporary Mathematical Sciences, vol. 7, no. 45, pp. 2179-2195, 2012.

[15] D. Wu and H. Zhang, "Bifurcation analysis of a two-species competitive discrete model of plankton allelopathy," Advances in Difference Equations, vol. 2014, no. 1, pp. 1-10, 2014.

[16] S. M. Rana, "Chaotic dynamics and control of discrete ratiodependent predator-prey system," Discrete Dynamics in Nature and Society, vol. 2017, Article ID 4537450, 13 pages, 2017.

[17] S. M. Rana and U. Kulsum, "Bifurcation analysis and chaos control in a discrete-time predator-prey system of Leslie type with simplified Holling type IV functional response," Discrete Dynamics in Nature and Society, vol. 2017, Article ID 9705985, 11 pages, 2017.

[18] J. Guckenheimer and P. Holmes, Nonlinear Oscillations, Dynamical Systems and Bifurcation of Vector Fields, SpringerVerlag, New York, NY, USA, 1983.

[19] Y. A. Kuznetsov, Elements of Applied Bifurcation Theorey, Springer-Verlag, New York, NY, USA, 3rd edition, 2004. 